\title{
Negative immersions for one-relator groups
}

\author{
Larsen Louder and Henry Wilton
}

May 7, 2021

\begin{abstract}
We prove a freeness theorem for low-rank subgroups of one-relator groups. Let $F$ be a free group, and let $w \in F$ be a non-primitive element. The primitivity rank of $w, \pi(w)$, is the smallest rank of a subgroup of $F$ containing $w$ as an imprimitive element. Then any subgroup of the onerelator group $G=F /\langle w\rangle\rangle$ generated by fewer than $\pi(w)$ elements is free. In particular, if $\pi(w)>2$ then $G$ doesn't contain any Baumslag-Solitar groups.

The hypothesis that $\pi(w)>2$ implies that the presentation complex $X$ of the one-relator group $G$ has negative immersions: if a compact, connected complex $Y$ immerses into $X$ and $\chi(Y) \geq 0$ then $Y$ Nielsen reduces to a graph.

The freeness theorem is a consequence of a dependence theorem for free groups, which implies several classical facts about free and one-relator groups, including Magnus' Freiheitssatz and theorems of Lyndon, Baumslag, Stallings and Duncan-Howie.

The dependence theorem strengthens Wise's w-cycles conjecture, proved independently by the authors and Helfer-Wise, which implies that the one-relator complex $X$ has non-positive immersions when $\pi(w)>1$.
\end{abstract}

\section{Introduction}

\subsection{One-relator groups}

The beginnings of combinatorial group theory are often identified with Dehn's articulation of the word, conjugacy and isomorphism problems [Deh11, and Magnus' solution of the word problem for one-relator groups was an early triumph of the subject Mag32. The contemporary approach to these decision problems takes the geometric route: to solve them in a class of groups $\mathcal{C}$, one first shows that the groups in $\mathcal{C}$ admit some kind of geometric structure. The fundamental example is the class of word-hyperbolic groups, for which the word, conjugacy and isomorphism problems have all been solved. Related techniques can be applied to handle other important classes: 3-manifold groups, sufficiently small-cancellation groups and fully residually free groups, to name a few.

After a century of progress, it is remarkable that the class of one-relator groups is still almost untouched by geometric techniques, and the conjugacy 
and isomorphism problems remain wide open. Many one-relator groups are word-hyperbolic - all one-relator groups with torsion, and a randomly chosen one-relator group is $C^{\prime}(1 / 6)$ - but there is also a menagerie of non-hyperbolic examples, including Baumslag-Solitar groups, Baumslag's example Bau69, fundamental groups of two-bridge knot complements, and the recent examples of Gardam-Woodhouse GW19].

In this paper, we present theorems about the structure of one-relator groups which begin to suggest a general geometric classification. A combinatorial map of 2-complexes $Y \rightarrow X$ is called an immersion if it is locally injective. The starting point for these results is a recent result established independently by the authors [WW17 and by Helfer-Wise HW16: the presentation complex $X$ of a torsion-free one-relator group has non-positive immersions, meaning that every connected, finite 2-complex $Y$ that immerses into $X$ either has $\chi(Y) \leq 0$ or Nielsen reduces ${ }^{1}$ to a point. We investigate the negatively curved analogue of this definition.

Definition 1.1. A compact 2-complex $X$ has negative immersions if, for every immersion from a compact, connected 2-complex $Y$ to $X$, either $\chi(Y)<0$ or $Y$ Nielsen reduces to a graph.

On the face of it, negative immersions should be a difficult condition to check, since it applies to all immersed compact complexes $Y$. However, there turns out to be a connection with a quantity defined by Puder Pud14.

Definition 1.2. Let $F$ be a free group and $w \in F$. The primitivity rank of $w$ is

$$
\pi(w)=\min \{\operatorname{rk}(K) \mid w \in K \leq F \text { and } w \text { not primitive in } K\} \in \mathbb{N} \cup\{\infty\},
$$

where, by convention, $\pi(w)=\infty$ if $w$ is primitive in $F$, since in that case $w$ is primitive in every subgroup $K$ containing $w$. Note that $\pi(1)=0$, since 1 is an imprimitive element of the trivial subgroup.

The first main theorem of this paper tells us that negative immersions for the presentation complex $X$ of a one-relator group $G=F /\langle\langle w\rangle$ are governed by $\pi(w)$.

Theorem 1.3 (Negative immersions for one-relator groups). The presentation complex of the one-relator group $F /\langle\langle w\rangle$ has negative immersions if and only if $\pi(w)>2$.

Thus, negative immersions can be determined in practice for the presentation complexes of one-relator groups. There is an algorithm to compute the primitivity rank $\pi(w)$ - see Lemma 6.4 - and furthermore it is often easy to compute it by hand for small examples by considering how a map representing $w$ factors through immersions.

\footnotetext{
${ }^{1}$ See Definition 6.7 for the definition of Nielsen reduction. For now it suffices to know that Nielsen reduction is stronger than homotopy equivalence .
} 
Example 1.4. Let $w=a c a b a^{-1} b^{-1} c^{-1} \in\langle a, b, c\rangle=F_{3}$. As usual, if $F_{3}$ is realised as the fundamental group of a bouquet $\Omega$ of 3 circles then $w$ can be represented by an immersion $S \rightarrow \Omega$ where $S$ is a graph homeomorphic to a circle. Represent a subgroup $K \leq F$ containing $w$ by a finite graph $\Gamma$ such that the map $w$ factors as

$$
S \leftrightarrow \Gamma \leftrightarrow \Omega .
$$

If the edges labelled by $a$ in $S$ are not all identified in $\Gamma$ then one such edge is only crossed once, which implies that $w$ is primitive in $K$. The same holds for $b$ and $c$, and this in turn implies that the map $\Gamma \rightarrow \Omega$ is the identity. Therefore $\pi(w) \geq 3$. Furthermore, the Whitehead graph of $w$ is connected without cut vertices, so $w$ is not itself primitive. Therefore $\pi(w)=3$.

The primitivity ranks of all words of length at most 16 in the free group of rank 4 were computed by Cashen-Hoffmann [CH20].

Puder [Pud15, Corollary 8.3] proved that a generic word $w$ in a free group of rank $k$ has $\pi(w)=k$, so in particular, when $\operatorname{rk} F>2$, a generic one-relator complex has negative immersions. Theorem 1.3 follows from Lemma 6.10, which is a finer classification of immersions from complexes with sufficiently large Euler characteristic.

Non-positive immersions constrains the subgroup structure of a group. Recall that a group $G$ is called coherent if every finitely generated subgroup is finitely presented. Non-positive immersions implies a homological version of coherence: if $X$ has non-positive immersions, then the second homology group of any finitely generated subgroup of $\pi_{1} X$ is finitely generated [LW17, Corollary 1.6]. Indeed, Wise conjectured that the fundamental groups of complexes with non-positive immersions are coherent. The authors and, independently, Wise, have shown that one-relator groups with torsion are coherent [WWar, Wis18.

Our next theorem asserts that negative immersions also constrain the subgroup structure of a one-relator group. Recall that a group $G$ is called $k$-free if every subgroup generated by $k$ elements is free. The rank of a group $G$ is the minimal number of elements needed to generate $G$, and is denoted $\operatorname{rk}(G)$. Note that, if $G$ has a one-relator presentation with $n$ generators, then either $G$ is free (of rank $n-1$ ) or $\operatorname{rk}(G)=n$; see Remark 6.15 below.

Theorem 1.5 (Low-rank subgroups of one-relator groups). Let $G=F /\langle w\rangle$ be a one-relator group with $\pi(w)>1$. There is a finite collection $P_{1}, \ldots, P_{n}$ of freely indecomposable, one-relator subgroups of $G$, each of rank $\pi(w)$, with the following property. Let $H \leq G$ be a finitely generated subgroup.

(i) If $\operatorname{rk}(H)<\pi(w)$ then $H$ is free.

(ii) If $\operatorname{rk}(H)=\pi(w)$ then $H$ is either free or conjugate into some $P_{i}$.

In particular, the one-relator group $G$ is $(\pi(w)-1)$-free.

The $P_{i}$ are defined in Subsection 6.1. Theorem 1.5 is a cousin of Magnus' Freiheitssatz, which says that if $H$ is a proper free factor of a free group $F$ and the natural map $H \rightarrow F /\langle w\rangle\rangle$ is not injective then $w$ is in fact conjugate into 
$H$ Mag30. Theorem 1.5 follows immediately from Lemma 6.16 which applies to homomorphisms from groups of low rank to $G$.

As far as the authors are aware, Theorem 1.5 implies all known $k$-freeness theorems for one-relator groups. For instance, combining Theorem 1.5 with [Pud15, Corollary 8.3] recovers the following theorem of Arzhantseva-Olshanskii AO96.

Corollary 1.6. A generic $k$-generator one-relator group is $(k-1)$-free.

Generic one-relator groups satisfy the $C^{\prime}(1 / 6)$ small-cancellation property. However, we emphasise that there are many words $w$ with $\pi(w)>2$ that are not small-cancellation, such as Example 1.4

Remark 1.7. It follows immediately from Theorem 1.5 that $\pi(w)$ is the minimal rank of a non-free subgroup of the one-relator group $G=F /\langle w\rangle\rangle$. In particular, $\pi(w)$ is an isomorphism invariant of $G$.

Taken together, Theorems 1.3 and 1.5 imply that one-relator groups with negative immersions have a similar subgroup structure to hyperbolic groups.

Corollary 1.8. Let $w$ be an element of a free group $F$. If the one-relator group $G=F /\langle w\rangle\rangle$ has negative immersions then $G$ doesn't contain any BaumslagSolitar groups and any finitely generated abelian subgroup of $G$ is cyclic.

A famous question in geometric group theory asks whether or not a group with a finite classifying space and without Baumslag-Solitar subgroups must be hyperbolic [Bes, Question 1.1]. Lyndon's identity theorem implies that presentation complexes of torsion-free one-relator groups are classifying spaces, so in light of Corollary 1.8 , the case of one-relator groups with negative immersions is of immediate interest.

Conjecture 1.9. Every one-relator group with negative immersions is hyperbolic.

A positive resolution of Conjecture 1.9 would resolve the conjugacy and isomorphism problems for the class of one-relator groups with negative immersions. Of course, one can also ask whether one-relator groups with negative immersions have other conjectural properties of hyperbolic groups, such as residual finiteness and surface subgroups.

Since $\pi(w)=1$ if and only if the corresponding one-relator group has torsion, and these are known to be hyperbolic by the B. B. Newman Spelling Theorem [New68, HW01, the remaining case of interest is $\pi(w)=2$. Onerelator groups with primitivity rank two seem to behave differently than the rest; we state a mild strengthening of Theorem 1.5 in this case.

Corollary 1.10. Let $w$ be an element of a free group $F$. If $\pi(w)=2$ then the one-relator group $G=F /\langle w\rangle$ contains a subgroup $P \leq G$ with the following properties:

(i) $P$ is a two-generator, one-relator group; 
(ii) every two-generator subgroup of $G$ is either free or conjugate into $P$.

We call the subgroup $P$ the peripheral subgroup of $G$ (we cannot currently prove that $P$ is an isomorphism invariant of $G$ ). We are unable to say anything new about two-generator one-relator groups - note that, in this case, Corollary 1.8 is vacuous and Corollary 1.10 is trivially true.

Example 1.11. Take $K$ to be a rank-two free factor $\langle x, y\rangle$ of a free group $F$, and let $w=[x, y]$. In this case, $G=F /\langle w\rangle\rangle$ is the free product of $P=K /\langle w\rangle\rangle \cong \mathbb{Z}^{2}$ together with a complementary free-group factor, and Corollary 1.10 implies that every freely indecomposable two-generator subgroup of $G$ is conjugate into $P$. In this case, the conclusion also follows from the Kurosh subgroup theorem; however, in more complicated examples, $G$ will not split as a free product and the Kurosh subgroup theorem will not apply.

Corollary 1.10 suggests the following natural counterpart to Conjecture 1.9

Conjecture 1.12. Suppose $\pi(w)=2$. Then $G$ is hyperbolic relative to $P$.

Conjectures 1.9 and 1.12 provide a conceptual explanation for the fact that all known examples of pathological one-relator groups have two generators.

\subsection{The dependence theorem}

In 1959, Lyndon proved that a non-trivial commutator in a free group $F$ cannot be expressed as a square [Lyn59. In this paper, we view Lyndon's theorem as the first in a line of dependence theorems for free groups, which bound the rank of the target of a homomorphism in which certain elements are forced either to be conjugate or to have roots.

Theorem (Lyndon, 1959). Let $H=\langle a, b\rangle, v=[a, b]$, and consider the group $G=H *_{v=x^{n}}\langle x\rangle$ where $n=2$. If $f: G \rightarrow F$ is a surjective homomorphism onto a free group then $\operatorname{rk}(F) \leq 1$.

Remark 1.13. Lyndon in fact proved this theorem for the word $v^{\prime}=a^{2} b^{2}$. This is equivalent to the theorem for $v=[a, b]$, by Dyck's theorem.

Shortly afterwards, the hypotheses of Lyndon's theorem were weakened to cover the case when $n \geq 2$; see, for example, Bau60, Lemma 36.4]. The commutator $v=[a, b]$ in Lyndon's theorem cannot be replaced by an arbitrary element of the free group; indeed, adjoining a root to a generator $a$ exhibits a map in which the rank of the target group does not go down. We therefore need a hypothesis that excludes generators. Recall that a collection of subgroups $\left\{M_{1}, \ldots, M_{n}\right\}$ of a group $H$ is called malnormal if $M_{i} \cap h M_{j} h^{-1} \neq 1$ implies that $i=j$ and $h \in M_{i}$, for any indices $i, j$ and $h \in H$.

Definition 1.14. A malnormal collection of cyclic subgroups $\left\{\left\langle v_{j}\right\rangle\right\}$ of a group $H$ is called independent if there exists a free splitting $H=H^{\prime} *\left\langle v_{k}\right\rangle$ of $H$, for some $k$, with $v_{j}$ conjugate into $H^{\prime}$ for $j \neq k$. Otherwise, $\left\{\left\langle v_{j}\right\rangle\right\}$ is called dependent. 
Note that a singleton $\{\langle v\rangle\}$ in a free group $H$ is dependent if and only if $v$ is not primitive. Using the theory of pro- $p$ groups, Baumslag generalized Lyndon's theorem to all dependent malnormal singletons $\{\langle v\rangle\}$ Bau65.

Theorem (Baumslag, 1965). Let $H$ be a free group, $\{\langle v\rangle\}$ dependent (i.e. not a primitive element) and malnormal (i.e. not a proper power) in $H$ and $n>1$. If $G=H *_{v=x^{n}}\langle x\rangle$ and $f: G \rightarrow F$ is a surjective homomorphism onto a free group, then $\operatorname{rk}(F)<\operatorname{rk}(H)$.

We now introduce the data for a more general dependence theorem. Let $H_{1}, \ldots, H_{l}$ be free groups and $\left\{\left\langle v_{i, j}\right\rangle\right\}_{i=1 \cdots l, j=1 \cdots m_{i}}$ a malnormal collection of non-trivial cyclic subgroups of $H_{i}$. For each $i$ and $j$, let $n_{i, j}$ be a positive integer. We associate a graph of groups $\Delta=\Delta\left(\left\{H_{i}\right\},\langle x\rangle,\left\{\left\langle v_{i, j}\right\rangle\right\},\left\{n_{i, j}\right\}\right)$ to these data as follows. There are $l$ vertices labelled by the $H_{i}$, arranged around one central vertex labelled $\langle x\rangle$. For each $i$ and $j$, there is an edge which attaches the subgroup $\left\langle v_{i, j}\right\rangle$ to the index- $n_{i, j}$ subgroup of the vertex group $\langle x\rangle$ via the homomorphism mapping $v_{i, j}$ to $x^{n_{i, j}} 2^{2}$

A dependence theorem relates these data to the rank of a possible free image of $\pi_{1}(\Delta)$. For instance, Lyndon's theorem is the case when $l=m=1, H=$ $\langle a, b\rangle, v=[a, b]$ and $n=2$. A more general theorem of this form can be proved using the techniques of [Lou13] (cf. Theorems 1.3 and 1.5 of that paper).

Theorem (Louder, 2013). Let $H_{1}, \ldots, H_{l}$ be free groups, $\left\{\left\langle v_{i, j}\right\rangle\right\}$ a malnormal collection of non-trivial cyclic subgroups of $H_{i}$ and $n_{i, j}$ positive integers. Let $\Delta$ be the associated graph of groups and let $f: \pi_{1}(\Delta) \rightarrow F$ be a surjective homomorphism to a free group with $\left.f\right|_{H_{i}}$ injective for each $i$. If the family $\left\{\left\langle v_{i, j}\right\rangle\right\}$ is dependent for each $i$, and $\sum_{i, j} n_{i, j}>1$, then

$$
\operatorname{rk}(F)-1<\sum_{i}\left(\operatorname{rk}\left(H_{i}\right)-1\right) .
$$

Baumslag's theorem, and hence Lyndon's, follows immediately. Indeed, if $\left.f\right|_{H}$ is not injective, the conclusion holds automatically, and otherwise the theorem applies. A 1983 theorem of Stallings in a similar spirit also follows Sta83a, Theorem 5.3]; we discuss Stallings' theorem in Subsection 5.1.

Another kind of dependence theorem constrains the integers $n_{i, j}$ in terms of the ranks of the $H_{i}$. A prototypical result here is provided by a theorem of Duncan and Howie, which extends and quantifies Lyndon's theorem by bounding from below the genus of a proper power DH91. A special case of the DuncanHowie theorem can be stated as follows.

Theorem (Duncan-Howie, 1991). Let $\Sigma$ be a compact, orientable surface of genus $g$ with one boundary component; let $H=\pi_{1}(\Sigma)$ and let $\langle v\rangle=\pi_{1}(\partial \Sigma)$. Let $\Delta$ be the graph of groups obtained by adjoining an nth root to $v$ (i.e. $\Delta=$ $\Delta(H,\langle x\rangle,\langle v\rangle, n))$ and $f: \pi_{1}(\Delta) \rightarrow F$ be a homomorphism onto a free group with $f(v) \neq 1$. Then $n \leq \operatorname{rk}(H)-1=2 g-1$.

\footnotetext{
${ }^{2}$ When $l=1$ or $m_{i}=1$ we will drop the indices $i$ or $j$ as appropriate, to minimize notation.
} 
Just as Lyndon's theorem was generalized from surfaces to more general dependent malnormal families of cyclic subgroups, so the Duncan-Howie theorem can be extended to arbitrary dependent malnormal families of cyclic subgroups. The following theorem, proved by the authors and also Helfer-Wise, answered Wise's w-cycles conjecture, which was made in connection with the question of whether or not one-relator groups are coherent HW16, LW17.

Theorem (Louder-Wilton, Helfer-Wise). Let $H$ be a free group, $\left\{\left\langle v_{j}\right\rangle\right\}$ a malnormal collection of non-trivial cyclic subgroups of $H$ and $n_{j}$ positive integers. Let $\Delta$ be the associated graph of groups and let $f: \pi_{1}(\Delta) \rightarrow F$ be a homomorphism to a free group with $\left.f\right|_{H}$ injective. If the family $\left\{\left\langle v_{j}\right\rangle\right\}$ is dependent then $\sum_{j} n_{j} \leq \operatorname{rk}(H)-1$.

Despite the fifty-nine years of work documented above, there are simple examples that do not fall within the scope of these theorems. For instance, consider the next example (which famously demonstrates that stable commutator length does not coincide with commutator length in free groups).

Example 1.15. Let $\Sigma$ be a torus with one boundary component. Let $F=\pi_{1} \Sigma=$ $\langle a, b\rangle$ and let $w=[a, b]$ correspond to the boundary component. Consider the homomorphism $F \rightarrow S_{3}$ given by $a \mapsto(23)$ and $b \mapsto(12)$, so $w \mapsto(123)$, and let $\Sigma^{\prime} \rightarrow \Sigma$ be the 3 -sheeted covering map corresponding to the stabiliser of 1 in $S_{3}$. The boundary component unwraps three times in $\Sigma^{\prime}$ and therefore, by computing Euler characteristic, $\Sigma^{\prime}$ is a surface of genus two with a single boundary component, represented by $v \in H=\pi_{1}\left(\Sigma^{\prime}\right)$.

In summary, in this example, $\operatorname{rk}(F)=2, \operatorname{rk}(H)=4,\langle u\rangle$ is dependent and malnormal in $H$, and the inclusion $H \rightarrow F$ sends $v \mapsto w^{3}$.

Example 1.15 is, of course, consistent with the theorems of Baumslag, the first author and Duncan-Howie. However, the first two theorems only assume that $v \mapsto w^{n}$ with $n>1$, and conclude that $\operatorname{rk}(F) \leq 3$. Likewise, the DuncanHowie theorem asserts that $n \leq 3$, but places no constraint on $\operatorname{rk}(F)$. Intuitively, one is lead to conjecture a common generalisation, which imposes an upper bound on $n+\operatorname{rk}(F)$.

Theorem 1.16. Let $H_{1}, \ldots, H_{l}$ be free groups, $\left\{\left\langle v_{i, j}\right\rangle\right\}$ a malnormal collection of non-trivial cyclic subgroups of $H_{i}$ and $n_{i, j}$ positive integers. Let $\Delta$ be the associated graph of groups and let $f: \pi_{1}(\Delta) \rightarrow F$ be a surjective homomorphism to a free group with $\left.f\right|_{H_{i}}$ injective for each $i$. Then

$$
\operatorname{rk}(F)-2+\sum_{i, j} n_{i, j} \leq \sum_{i}\left(\operatorname{rk}\left(H_{i}\right)-1\right)
$$

if the family $\left\{\left\langle v_{i, j}\right\rangle\right\}$ is dependent for each $i$.

We do not know if the inequality of Theorem 1.16 is sharp; see Question 2.23.

As stated, Theorem 1.16 does not strictly generalize the Duncan-Howie theorem, since the map $f$ in Theorem 1.16 is required to be injective on the $H_{i}$. Theorem 2.21 relaxes the injectivity hypothesis to a hypothesis of 'diagrammatic 
irreducibility', which is weak enough to encompass the Duncan-Howie theorem; see Corollary 5.8 for details.

The connection between the dependence theorem and one-relator groups goes via an estimate on the Euler characteristic of the one-relator pushout of a branched map; the reader is referred to Definitions 2.2 and 3.1 for the relevant terms. A special case of the estimate can be stated as follows, which is a direct consequence of Corollary 3.2 .

Corollary 1.17. Let $f: Y \rightarrow X$ be an immersion from a compact, connected two-complex $Y$ to the presentation complex $X$ of a one-relator group $G=$ $F /\langle\langle w\rangle$, with $w$ not a proper power. If $Y$ has no free faces then

$$
\chi(Y) \leq \chi(\hat{Y}),
$$

where $\hat{Y}$ is the one-relator pushout of $f$.

As well as the applications to non-positive immersions mentioned above, this estimate on Euler characteristics also gives new proofs of Magnus' Freiheitssatz and Lyndon's asphericity theorem; see Theorem 5.4 .

\subsection{Remarks about Theorem $\mathbf{1 . 1 6}$ and its proof}

The proof of Theorem 1.16 combines stackings (first defined in [LW17]) with adjunction spaces (the main tool of [Lou13]). The definition of a stacking was inspired by the proof of the Duncan-Howie theorem, which in turn draws on the theory of one-relator groups developed by Magnus and others, as well as the tower argument of Papakyriakopoulos.

The adjunction space is the natural topological representative of the graph of groups $\Delta$ considered in the previous section. The map $\pi_{1}(\Delta) \rightarrow F$ can be realised by a second graph-of-spaces structure on the adjunction space, and the rank of the underlying graph of gives an upper bound for $\operatorname{rk}(F)$ - see Remark 2.10. In a nutshell, the idea of the proof is now to use the stacking constructed in LW17 to analyse the homology of the adjunction space in a manner reminiscent of Morse theory. The stacking enables us to define fibrewise filtrations on the adjunction space. An analysis of these filtrations reduces the proof of the main theorem to a combinatorial lemma - the up-down lemma of $\$ 4.4$.

The resulting inequality, that of Theorem 1.16, combines the degrees of the adjunctions with the rank of the target. This improves on both the DuncanHowie theorem (and its generalisation in [LW17]), which only sees the degrees of the adjunctions, and the theorems of Baumslag, Stallings and the first author, which only see the rank of the target. Morally, Theorem 1.16 can be thought of as a kind of non-abelian rank-nullity theorem.

\section{Acknowledgements}

The second author was funded by EPSRC Standard Grant EP/L026481/1. The authors are grateful to the anonymous referees for their close reading of the 
manuscript; their comments greatly improved the exposition. The authors are also grateful to Jim Howie and Hamish Short for pointing out an error in the the original version of Lemma 6.9

\section{Graphs and graphs of graphs}

\subsection{Graphs}

We start by recalling the basic set-up of graphs.

An (oriented) graph $G$ is a tuple $G=\left(V_{G}, E_{G}, \iota, \tau\right)$, where $V_{G}$ and $E_{G}$ are sets, (called the vertices and edges of $G$, respectively) and $\iota: E_{G} \rightarrow V_{G}$ and $\tau: E_{G} \rightarrow V_{G}$ are maps (called incidence maps). When convenient we suppress the subscript $G$. We often use the letter $\alpha$ to denote an incidence map, which might be either $\iota$ or $\tau$.

A morphism of graphs is a pair of maps $f: V_{G} \rightarrow V_{G^{\prime}}$ and $f: E_{G} \rightarrow E_{G^{\prime}}$, such that the natural diagrams

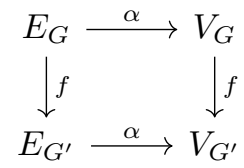

commute, for $\alpha=\iota, \tau$. A graph is simple if its edges are determined by their endpoints, i.e., if $\iota(e)=\iota\left(e^{\prime}\right)$ and $\tau(e)=\tau\left(e^{\prime}\right)$ then $e=e^{\prime}$. Note that in this case, the map $(\iota, \tau)$ naturally identifies $E_{G}$ with a subset of $V_{G} \times V_{G}$.

If there is a partition $V_{G}=I_{G} \sqcup T_{G}$ such that $\iota\left(E_{G}\right) \subseteq I_{G}$ and $\tau\left(E_{G}\right) \subseteq T_{G}$ then $G$ is called bipartite. A morphism of bipartite graphs is a morphism of graphs that respects the bipartite structure. Again, if $G$ is a simple bipartite graph, then $(\iota, \tau)$ identifies $E_{G}$ with a subset of $I_{G} \times T_{G}$.

Given a graph $G$ the geometric realization of $G$ is the 1-complex

$$
\boldsymbol{G}=\left(V_{G} \sqcup\left(E_{G} \times[-1,1]\right)\right) /\{(e,-1) \sim \iota(e),(e, 1) \sim \tau(e)\} .
$$

We implicitly identify $V_{G}$ with its image in $\boldsymbol{G}$.

The realization of a morphism of graphs $f: G \rightarrow G^{\prime}$ is the map

$$
\boldsymbol{f}(x)= \begin{cases}f(x) & \text { if } x \in V_{G} \\ (f(e), t) & \text { if } x=(e, t) \in e \times[-1,1]\end{cases}
$$

The (Euler) characteristic of a graph $G$ is defined to be quantity

$$
\chi(G):=\left|V_{G}\right|-\left|E_{G}\right| .
$$

For a choice of $v_{0} \in V_{G}$, we define $\pi_{1}\left(G, v_{0}\right):=\pi_{1}\left(\boldsymbol{G}, v_{0}\right)$. As usual, we will suppress mention of the base point $v_{0}$ when it will not cause confusion. In the usual way, a morphism of graphs $f: G \rightarrow G^{\prime}$ induces a homomorphism $f_{*}=\boldsymbol{f}_{*}$ on fundamental groups. 
The valence of a vertex $v \in V_{G}$ is defined to be

$$
\operatorname{val}(v):=\#\left\{e \in E_{G} \mid \iota(e)=v\right\}+\#\left\{e \in E_{G} \mid \tau(e)=v\right\} .
$$

If $\boldsymbol{G}$ is homeomorphic to $S^{1}$ then we say that $G$ is a cycle; equivalently, $G$ is finite and connected, and every vertex has valence two.

A morphism of graphs is an immersion if for all $e \neq e^{\prime}$ and $\alpha \in\{\iota, \tau\}$, $\alpha(e)=\alpha\left(e^{\prime}\right)$ implies $f(e) \neq f\left(e^{\prime}\right)$. Note that the realization $\boldsymbol{f}$ of an immersion $f$ is locally injective, and by Stallings [Sta83b, 5.3] induces an injective map $f_{*}$ on fundamental groups.

As in Sta83b, a finite graph is called a core graph if there are no vertices of valence 1 .

\subsection{Combinatorial complexes}

We are now ready to define the class of 2-complexes that we will work with.

Definition 2.1. A combinatorial (2-dimensional) complex $X$ is a tuple

$$
\left(G_{X}, S_{X}, w_{X}, o_{X}\right)
$$

where $G_{X}$ is a graph, $S_{X}$ is a disjoint union of cycles, $w_{X}: S_{X} \rightarrow G_{X}$ is an immersion of graphs and $o_{X}$ is an orientation on $\boldsymbol{S}_{X}$. We emphasise that $o_{X}$ is not required to relate to the structure of $S_{X}$ as an oriented graph, and in general, it will not.

As usual, we suppress subscripts when it will not cause confusion. We will also often suppress mention of the orientation $o_{X}$ as well.

A morphism of combinatorial complexes $f: X \rightarrow X^{\prime}$ consists of a map of graphs $f: G_{X} \rightarrow G_{X^{\prime}}$ and an immersion $s: S_{X} \rightarrow S_{X^{\prime}}$ such that the diagram

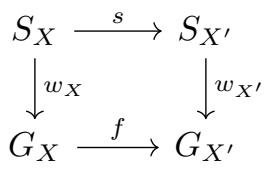

commutes and $s^{*}\left(o_{X^{\prime}}\right)=o_{X}$. We emphasise that, to avoid the notation becoming too burdensome, we will usually use the same letter to denote the map of 2-complexes and the the map of 1-skeleta, and introduce the letter $s$ to denote the accompanying map of circles.

The realization of a combinatorial complex $X$ is the space

$$
\boldsymbol{X}:=\boldsymbol{G}_{X} \sqcup\left(\boldsymbol{S}_{X} \times[0,1]\right) / \sim
$$

where $(\theta, 0) \sim \boldsymbol{w}(\theta)$ for all $\theta \in \boldsymbol{S}_{X}$ and $\left(\theta_{1}, 1\right) \sim\left(\theta_{2}, 1\right)$ whenever $\theta_{1}$ and $\theta_{2}$ are in the same component of $\boldsymbol{S}_{X}$. Note that this definition is functorial: a morphism $f$ of combinatorial complexes $X \rightarrow X^{\prime}$ naturally induces a continuous map $\boldsymbol{f}: \boldsymbol{X} \rightarrow \boldsymbol{X}^{\prime}$. As usual, given a choice of vertex $x_{0}$ in $G_{X}$, we define $\pi_{1}\left(X, x_{0}\right):=\pi_{1}\left(\boldsymbol{X}, x_{0}\right)$. 
A free face of a combinatorial complex $X$ is an edge $e \in E_{G_{X}}$ such that $\left|w_{X}^{-1}(e)\right|=1$. Note that if $X$ has a free face, then $\boldsymbol{X}$ can be simplified by a simple homotopy, which collapses a 2-cell (see Section 6.2). The boundary of $X, \partial X$, is the union of its free faces. The boundary of the realization, $\partial \boldsymbol{X}$, is the union of the closed edges corresponding to $\partial X$.

The link of a vertex $v$ of $G_{X}$ is a graph $L \equiv L(v)$ defined as follows. The set of vertices of $L$ is

$$
V_{L}:=\left\{(x, \alpha) \in E_{G_{X}} \times\{\iota, \tau\} \mid \alpha(x)=v\right\}
$$

and the set of edges is $E_{L}:=w^{-1}(v) \subseteq V_{S_{X}}$. Since $S_{X}$ is a disjoint union of cycles, for each $y \in E_{L}$ there exist exactly two edges of $E_{S_{X}}$ incident in $S_{X}$ at $y$. Let $i_{y}$ be the edge immediately preceding $y$ according to $o_{X}$ and let $\alpha_{y} \in\{\iota, \tau\}$ such that $\alpha_{y}\left(i_{y}\right)=y$. Likewise, let $t_{y}$ be the edge immediately following $y$ according to $o_{X}$ and let $\beta_{y}\left(t_{y}\right)=y$. Then $\left(w\left(i_{y}\right), \alpha_{y}\right)$ and $\left(w\left(t_{y}\right), \beta_{y}\right)$ are both vertices of $V_{L}$, and we define the incidence maps of $L$ by setting $\iota(y)=$ $\left(w\left(i_{y}\right), \alpha_{y}\right)$ and $\tau(y)=\left(w\left(t_{y}\right), \beta_{y}\right)$.

A morphism of combinatorial complexes $f: X \rightarrow X^{\prime}$ naturally induces maps on links $f_{v}: L(v) \rightarrow L(f(v))$.

We can now define the class of morphisms that we are concerned with. Informally, branched maps are maps that are locally injective away from vertices and midpoints of 2-cells, and immersions are locally injective everywhere.

Definition 2.2. A morphism $f: X \rightarrow X^{\prime}$ of combinatorial complexes is a branched map if every induced map on links $f_{v}$ is an immersion. Furthermore, if every $f_{v}$ is injective and the map $s: S_{X} \rightarrow S_{X^{\prime}}$ is injective on each component, we say that $f$ is an immersion. In this case, we write $f: X \rightarrow X^{\prime}$.

In combinatorial group theory, a special role is played by van Kampen diagrams - planar 2-complexes that represent relations in the fundamental group. These can be seen as special cases of morphisms of combinatorial complexes.

Definition 2.3. Let $X$ be a combinatorial complex. A van Kampen diagram over $X$ is pair of morphisms

$$
S \rightarrow D \rightarrow X
$$

where $S$ is a cycle and $\boldsymbol{D} \cup_{S} D^{2}$ is homeomorphic to the 2-sphere $S^{2}$. If $S \rightarrow D$ is an immersion and $D \rightarrow X$ is a branched map then the van Kampen diagram is said to be reduced.

Remark 2.4. This notion of van Kampen diagram is slightly more general than the standard one (cf. LS01, Chapter III, §9]), since we allow branching over the centres of the 2-cells in $D$.

The next lemma provides a useful characterization of branched maps.

Lemma 2.5. A morphism $f$ of combinatorial complexes

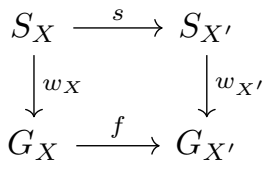


is a branched map if and only if the map

$$
\left(w_{X}, s\right): E_{S_{X}} \rightarrow E_{G_{X}} \times E_{S_{X^{\prime}}}
$$

is an embedding.

Proof. The link of an edge $e$ in a combinatorial complex $X$ is the set $w_{X}^{-1}(e)$. For any vertex $v$ of $X$, the map $f_{v}: L(v) \rightarrow L(f(v))$ is an immersion if and only if it does not fold any pair of edges of $L(v)$. Equivalently, $f_{v}$ is an immersion if and only if $s$ restricts to an injective map on the star of every vertex of $L(v)$. Therefore, $f$ is a branched map if and only if it induces injective maps on the links of edges of $X$. To complete the proof of the lemma, note that $s$ is injective on each link $w_{X}^{-1}(e)$ if and only if the map $\left(w_{X}, s\right)$ is injective.

\subsection{Graphs of graphs and the adjunction space}

The construction below appears in various guises in the papers [Dic94, LM09, Lou13.

Definition 2.6. A graph of graphs is a graph $M=\left(V_{M}, E_{M}, \varphi, \psi\right)$, where the vertex and edge sets $V_{M}$ and $E_{M}$ are collections of graphs. The elements of $V_{M}$ are the vertex graphs of $M$, and the elements of $E_{M}$ are the edge graphs of $M$. The incidence maps $\varphi$ and $\psi$ are considered as maps from $V_{M}$ to $E_{M}$ as sets, but which specify morphisms of graphs. That is, given $E \in E_{M}$ there are morphisms of graphs $\varphi_{E}: E \rightarrow \varphi(E) \in V_{M}$ and $\psi_{E}: E \rightarrow \psi(E) \in V_{M}$.

Given a graph of graphs $M$, we construct a new graph $\Gamma_{M}$, which is the graph $M$ with the additional data forgotten. There is a natural "forgetful" (iso)morphism of graphs $\pi: M \rightarrow \Gamma_{M}$. For each $x \in E_{\Gamma_{M}} \sqcup V_{\Gamma_{M}}$, we let $M_{x}$ denote the graph $\pi^{-1}(x)$. In what follows we keep this notation. Furthermore, if $M_{e} \in E_{M}$ is an edge graph then $\varphi\left(M_{e}\right) \subseteq M_{v} \in V_{M}$, for some $v=\varphi(e) \in V_{\Gamma_{M}}$, and to avoid unnecessary subscripts, we denote the map $\varphi_{M_{e}}$ simply by $\varphi$. Likewise for $\psi$. In this way we think of $M$ as being a family of graphs "indexed" by the graph $\Gamma_{M}$, which is just the graph of graphs $M$ with its additional structures stripped away.

A morphism of graphs of graphs $f: M \rightarrow M^{\prime}$ is a morphism of graphs, where the map $f$ is endowed with additional structures compatible with the graph of graphs structure, i.e., for each $x \in \Gamma_{M}$, there is a morphism of graphs $f_{x}: M_{x} \rightarrow M_{f(x)}$ which is compatible with edge maps. That is, for each $e \in E_{\Gamma_{M}}$ the following squares commute.
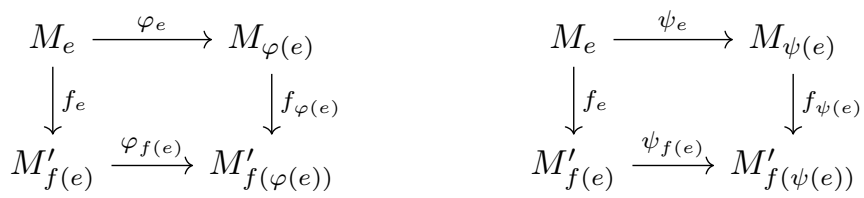

The set of vertex graphs $V_{M}$ is the collection of graphs $\left\{M_{v}\right\}_{v \in V_{\Gamma_{M}}}$, and we abuse notation and denote the disjoint union $\coprod M_{v}$ by $M_{V}$. Likewise for edge 
graphs. The realization $\boldsymbol{M}$, defined below, has an additional graph of graphs structure $M^{\prime}=\left(\left\{M_{V}\right\},\left\{M_{E}\right\}, \varphi, \psi\right)$ with one vertex space and one edge space, i.e., the underlying graph $\Gamma_{M^{\prime}}$ has only one vertex and one edge, with edge maps $\varphi$ and $\psi$. Clearly $\boldsymbol{M}^{\prime}$ is naturally homeomorphic to $\boldsymbol{M}$. This additional graph of graphs structure will turn up again in Subsection 4.2, where it is used in a Mayer-Vietoris argument.

Definition 2.7. The realization of a graph of graphs $M$ is the space

$$
\boldsymbol{M}:=\boldsymbol{M}_{V} \sqcup\left(\boldsymbol{M}_{E} \times[-1,1]\right) / \sim
$$

where $(x,-1) \sim \varphi(x)$ and $(x,+1) \sim \psi(x)$ for all $x \in M_{E}$.

The notion of realization allows us to define fundamental groups and other topological invariants of $M$ as usual. The Euler characteristic will play a particularly important role.

Definition 2.8. The (Euler) characteristic of a graph of graphs $M$ is

$$
\chi(M):=\chi\left(M_{V}\right)-\chi\left(M_{E}\right) .
$$

Note that $\chi(M)$ is the Euler characteristic of $\boldsymbol{M}$.

Remark 2.9. Any oriented graph $G=\left(V_{G}, E_{G}, \iota, \tau\right)$ can be regarded as a graph of graphs by regarding $V_{G}$ and $E_{G}$ as graphs with no edges, and regarding the edge maps $\iota$ and $\tau$ as morphisms of graphs. In this case the indexing graph has one edge and one vertex, with edge maps $\iota$ and $\tau$. In this case the forgetful map returns the graph itself. Likewise, a bipartite graph may be regarded as a graph of graphs indexed by a graph with two vertices and one edge.

We are particularly interested in graphs of graphs with connected vertex and edge spaces. Given a graph of graphs $M$, there is a canonical $\hat{M}$ with connected vertex and edge spaces, and a morphism $f: \hat{M} \rightarrow M$ with the following property: For each $y \in \Gamma_{M}$, the restriction

$$
f: \coprod_{x \in f^{-1}(y)} \hat{M}_{x} \rightarrow M_{y}
$$

is an isomorphism of graphs. The vertex graphs of $\hat{M}$ are the connected components of the vertex graphs of $M$, the edge graphs of $\hat{M}$ are the connected components of the edge graphs of $M$, and the edge maps of $\hat{M}$ are induced by restriction. Furthermore, the realisation $f: \hat{M} \rightarrow \boldsymbol{M}$ is a homeomorphism.

Therefore, without loss of generality, we may always assume that graphs of graphs have connected vertex and edge spaces, and we will do so.

Remark 2.10. If the vertex and edge graphs of $M$ are connected then the natural $\operatorname{map} \pi_{1}(M) \rightarrow \pi_{1}\left(\Gamma_{M}\right)$ is surjective.

In the terminology of Wise and his coauthors, the realization $M$ is a $V H$ complex BW99, and the maps $\varphi$ and $\psi$ are the incidence maps of the vertical 
graph-of-graphs structure on $\boldsymbol{M}$. We now turn our attention to the equally natural horizontal graph-of-graphs structure on $\boldsymbol{M}$, called $W$.

Let $\iota$ and $\tau$ be the incidence maps coming from the defining data of the graphs in $V_{M}$ and $E_{M}$. We define a new intermediate graph of graphs structure, $N$, as follows: $N$ has one vertex graph $V$ with vertex set $\sqcup_{v \in V_{\Gamma_{M}}} V_{M_{v}}$ and edge set $\sqcup_{e \in E_{\Gamma_{M}}} V_{M_{e}}$, with edge maps induced by $\varphi$ and $\psi$, and $N$ has one edge graph $E$ with vertex set $\sqcup_{v \in V_{\Gamma_{M}}} E_{M_{v}}$ and edge set $\sqcup_{e \in E_{\Gamma_{M}}} E_{M_{e}}$, with edge maps again induced by $\varphi$ and $\psi$. The collection of incidence maps $\tau$ and $\iota$ join together to induce maps $\tau: E \rightarrow V$ and $\iota: E \rightarrow V$. We then set $W=\hat{N}$. The next lemma records the fact that this is an alternative graph of graphs structure on $\boldsymbol{M}$.

Lemma 2.11. The realizations $\boldsymbol{W}$ and $\boldsymbol{M}$ are homeomorphic.

Remark 2.12. It is the fact that our graphs and their morphisms are oriented that automatically endows $\boldsymbol{M}$ with the dual graph-of-graphs decomposition. This need not hold for graphs of unoriented graphs.

Remark 2.13. The underlying graph $\Gamma_{W}$ is the pushout of the diagram

$$
\amalg M_{e} \stackrel{\varphi}{\longrightarrow} \amalg M_{v}
$$

in the category of (oriented) graphs.

A morphism of combinatorial complexes naturally defines a graph of graphs - the adjunction space.

Definition 2.14. Let $f: X \rightarrow X^{\prime}$ be a morphism of combinatorial complexes. The adjunction space $M \equiv M(f)$ is the (bipartite) graph of graphs with vertex set

$$
V_{M}:=\left\{G_{X}, S_{X^{\prime}}\right\},
$$

edge set

$$
E_{M}:=\left\{S_{X}\right\}
$$

and incidence maps given by $s: S_{X} \rightarrow S_{X}^{\prime}$ and $w: S_{X} \rightarrow G_{X}$.

\subsection{Resolving}

We now specialize the discussion above to the case of interest for our main theorem. We start with two combinatorial complexes and a map $h$ between them, defined by the following data.

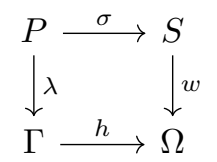

Let $M$ be the adjunction space of $h$ and let $W$ be the horizontal graph of graphs associated with $M$. We now observe that this set-up entails the existence of various natural maps, summarized in the following commutative diagram. 


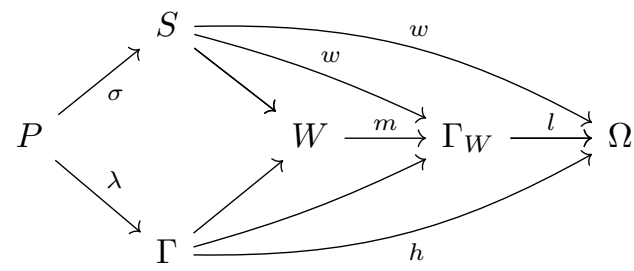

The morphism $h$ determines a map of graph $\AA^{3} W \rightarrow \Omega$ that sends vertical vertex-graphs to vertices and vertical edge-graphs to midpoints of edges.

We now resolve this map, by factoring it through the underlying graph $\Gamma_{W}$ of $W$. The map $W \rightarrow \Omega$ factors through the natural map $m: W \rightarrow \Gamma_{W}$. There are natural morphisms $S \rightarrow W$ and $\Gamma \rightarrow W$ which, when composed with $m$, descend to morphisms $S \rightarrow \Gamma_{W}$ and $\Gamma \rightarrow \Gamma_{W}$. By Remark 2.13, the maps $S \rightarrow \Omega$ and $\Gamma \rightarrow \Omega$ factor through a canonical map of graphs $l: \Gamma_{W} \rightarrow \Omega$; the map of graphs $W \rightarrow \Omega$ then factors as:

$$
W \stackrel{m}{\longrightarrow} \Gamma_{W} \stackrel{l}{\longrightarrow} \Omega .
$$

For the most part in what follows, this enables us to replace $\Omega$ by $\Gamma_{W}$, and for that reason, we will also denote by $w$ the natural map $S \rightarrow \Gamma_{W}$, even though, strictly speaking $w$ is a map from $S$ to $\Omega$.

We denote by $\Gamma_{W}^{I}$ the graph obtained by Stallings folding the map $l: \Gamma_{W} \rightarrow$ $\Omega$ to an immersion. Note that $\chi\left(\Gamma_{W}^{I}\right) \geq \chi\left(\Gamma_{W}\right)$.

Remark 2.15. We can use this set-up to draw group-theoretic conclusions about $\pi_{1}(\Omega)$, since the induced homomorphism $\pi_{1}(\boldsymbol{W}) \rightarrow \pi_{1}(\boldsymbol{\Omega})$ factors through the map $\boldsymbol{m}_{*}: \pi_{1}(\boldsymbol{W}) \rightarrow \pi_{1}\left(\boldsymbol{\Gamma}_{W}\right)$. Note that point preimages in $\boldsymbol{m}$ are connected, and therefore $\boldsymbol{m}_{*}$ is surjective.

\subsection{The dependence theorem}

The boundary of $W$ consists of those edges of $\Gamma$ that are hit by precisely one element of $P$.

Definition 2.16. Let $W$ be the horizontal graphs of graphs for the adjunction space defined above. The boundary of $W$ is

$$
\partial W=\left\{e \in E_{\Gamma}|| \lambda^{-1}(e) \mid=1\right\} .
$$

The boundary of $\boldsymbol{W}$ is

$$
\partial \boldsymbol{W}=\bigcup_{e \in \partial W} \overline{e \times(-1,1)} \subseteq \boldsymbol{\Gamma} .
$$

By construction $\partial W$ is the boundary of the complex defined by $\lambda: P \rightarrow \Gamma$. As mentioned above, when $W$ has nonempty boundary, this complex can be

\footnotetext{
${ }^{3}$ But not of graphs of graphs.
} 


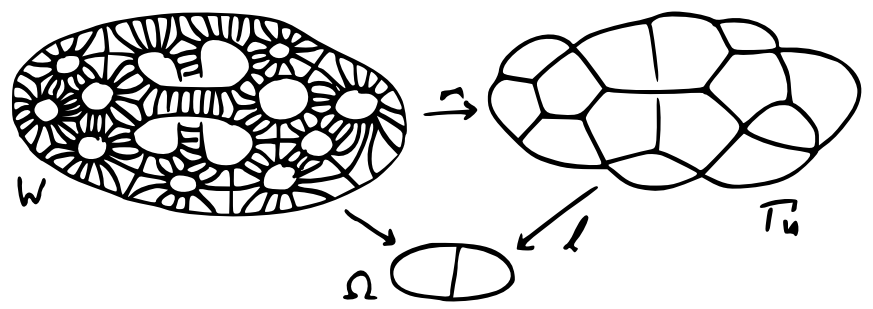

Figure 1: Schematic of $W$. The realization $\boldsymbol{W}$ is the graph of spaces obtained by gluing the ends of $\boldsymbol{P} \times I$ to $\boldsymbol{\Gamma}$ and $\boldsymbol{S}$ using $\lambda$ and $\sigma$. We think of $\boldsymbol{\Gamma}$ and $\boldsymbol{S}$ as running through $\boldsymbol{W}$ horizontally. The vertical graph-of-graphs structure on $\boldsymbol{W}$ is cartoonishly depicted above, with vertex spaces $\boldsymbol{W}_{v}$ and edge spaces $\boldsymbol{W}_{e}$. The morphism of graphs $W \rightarrow \Omega$ factors as $l \circ m$, where $m$ is the projection from $W$ to its underlying graph $\Gamma_{W}$.

simplified by a collapse. We call this circumstance independent (since it implies the group-theoretic notion of independence given in the introduction). We will also be interested in a strengthening of this, in which the whole image of $S$ in $\Gamma_{W}$ (and therefore in $\Omega$ ) is covered at least twice by the boundary.

Definition 2.17. The map $\lambda: P \rightarrow \Gamma$ is independent if $\partial W \neq \varnothing$; otherwise, it is called dependent. The map $\lambda: P \rightarrow \Gamma$ is strongly independent (over $\Omega$ ) if, for all $e \in w\left(E_{S}\right),\left|\partial W \cap W_{e}\right| \geq 2$; otherwise, it is called weakly dependent (over $\Omega$ ).

Example 2.18. Let $\Omega$ be the (oriented) graph with one vertex $v$ and two edges $a$ and $b$, and let $X$ be the combinatorial complex $(\Omega, S, w, o)$, where $S$ is a cycle with three edges, $w: S \rightarrow \Omega$ is the immersion determined by the word $a b b$, and $o$ is arbitrary. The realization of $X$ is the Möbius strip, with one boundary edge labeled $a$. Let $Y=\left(\Gamma, P, \lambda, o^{\prime}\right)$ be the combinatorial complex corresponding to the annular connected double cover of $X$ with the two lifts of the vertex identified (with $o^{\prime}$ pulled back from $o$ ). Then the boundary of $W$ consists of two edges which both map to the edge $a$ in $\Omega$, so $\partial W$ does not (doubly) surject $w\left(E_{S}\right)=E_{\Omega}$, and in this case $\lambda$, which represents the attaching map for the two two-cells in $Y$, is weakly dependent. It is not, however dependent, since the boundary is non-empty.

We are interested in the setting where $h$ is a branched map. This has the following consequences for $W$.

Lemma 2.19. Let $W$ be the horizontal graphs of graphs for the adjunction space associated to a branched map $h$.

(i) The graph $W_{E}$ is a simple bipartite graph.

(ii) The incidence maps of $W$ are injective on the edges of each edge space $W_{e}$, and also on the set of vertices of $W_{e}$ that come from $S$. 
The proof is left as an easy exercise.

Remark 2.20. If $S$ is connected and $\sigma: P \rightarrow S$ is a covering map then, for each $s \in S_{x} \subset W_{x}, \operatorname{val}(s)=\operatorname{deg}(\sigma)$.

In our case, the complex defined by the map $w: S \rightarrow \Omega$ will be a one-relator complex, meaning that $S$ is just a single cycle. We call $w$ indivisible if it does not factor through a proper covering map $S \rightarrow S^{\prime}$.

We can now state the dependence theorem in the form in which we prove it.

Theorem 2.21 (Dependence theorem). Let $h$ be a branched map of combinatorial complexes as above, and let $W$ be the horizontal graph of spaces for the adjunction space. Suppose further that $S$ is a single cycle and that $w: S \rightarrow \Omega$ is indivisible. If $\lambda: P \rightarrow \Gamma$ is weakly dependent then

$$
\chi(\Gamma)+\operatorname{deg}(\sigma)-1 \leq \chi\left(\Gamma_{W}\right) .
$$

Usually, following Sta83b, subgroups of free groups are represented by immersions of connected graphs, so for the purposes of generalizing the theorems of Baumslag and Stallings it is safe to restrict to immersions of connected $\Gamma \rightarrow \Omega$. However, in order to strengthen the Duncan-Howie theorem we need to allow maps that are not immersions.

Example 2.22. Theorem 2.21 does not hold when the condition that $w$ be indivisible is relaxed, as the following example illustrates. Let $\Omega$ be the graph with one vertex $v$ and one one-cell $e$, let $h: \Gamma \rightarrow \Omega$ and $w: S \rightarrow \Omega$ be the connected degree-two and degree-three covers, respectively, and let $P \rightarrow \Omega$ be the connected degree-six cover. Let $\lambda: P \rightarrow \Gamma$ be the degree-three cover and $\sigma: P \rightarrow S$ a degree-two cover. In this example, $\Gamma_{W} \cong \Omega$, and $\lambda$ is weakly dependent, since each vertex and edge graph $W_{e}$ and $W_{v}$ is isomorphic to the complete bipartite graph $K_{2,3}$, but the theorem predicts

$$
0+2-1 \leq 0
$$

which is, of course, false.

If $h$ is a branched map and $\lambda$ is weakly dependent then $\chi\left(\Gamma_{W}\right) \leq-1$, and in this case Theorem 2.21 implies the inequality

$$
\chi(\Gamma)+\operatorname{deg}(\sigma) \leq 0,
$$

which is precisely Wise's $w$-cycles conjecture [HW16, LW17.

Since we do not know if the inequality of Theorem 2.21 is sharp, we pose the question here.

Question 2.23. Are there $W$ as above, with $\lambda$ dependent, such that

$$
\chi(\Gamma)+\operatorname{deg}(\sigma)-1=\chi\left(\Gamma_{W}\right)
$$

for all $\operatorname{deg}(\sigma) \geq 2$ and $\chi\left(\Gamma_{W}\right) \leq-1$ ? What about for $\lambda$ weakly dependent?

We next explain how Theorem 2.21 implies Theorem 1.16 . 
Proof of Theorem 1.16. We may assume that $f(w)$ is not a proper power in $F$ : if there are $v \in F, k \geq 1$, such that $f(w)=v^{k}$ then, since $f$ is surjective, if

$$
\operatorname{rk}(F)-2+\sum_{i, j} k n_{i, j} \leq \sum_{i}\left(\operatorname{rk}\left(H_{i}\right)-1\right),
$$

then certainly

$$
\operatorname{rk}(F)-2+\sum_{i, j} n_{i, j} \leq \sum_{i}\left(\operatorname{rk}\left(H_{i}\right)-1\right) .
$$

We take $\Omega$ to be a rose with $F=\pi_{1}(\Omega)$ a free group, $\Gamma$ to be a graph immersing into $\Omega$, for which the components have fundamental groups $H_{i}$, and $\lambda: P \rightarrow \Gamma$ an immersion of a disjoint union of cycles into $\Gamma$ that represent the family $\left\{v_{i, j}\right\}$. Since each $f\left(v_{i, j}\right)$ is conjugate into $\langle w\rangle$, these factor through a common cycle $w: S \rightarrow \Omega$ which induces the maps $\sigma: P \rightarrow S$ and $\lambda: P \rightarrow \Gamma$. We may therefore construct the adjunction space $M$ and its associated horizontal graph of graphs $W$.

By definition, $\pi_{1}(\Delta)=\pi_{1}(M)$, which is in turn canonically isomorphic to $\pi_{1}(W)$ by Lemma 2.11. The map $\pi_{1}(W) \rightarrow F$ is surjective by Remark 2.10 and factors through the surjection $m_{*}: \pi_{1}(W) \rightarrow \pi_{1}\left(\Gamma_{W}\right)$ so $\chi(F)=\chi(\Omega) \geq \chi\left(\Gamma_{W}\right)$.

Because $\left\{\left\langle v_{i, j}\right\rangle\right\}$ is dependent, it follows that the map $\lambda: P \rightarrow \Gamma$ is dependent, in particular weakly dependent. Since $\left\{\left\langle v_{i, j}\right\rangle\right\}$ is malnormal, the natural map $P \rightarrow \Gamma \times \Omega$ is an embedding, so $(\Gamma, P) \rightarrow(\Omega, S)$ is a branched map of 2 -complexes, by Lemma 2.5. The result now follows from Theorem 2.21, after noting that

$$
\operatorname{deg}(\sigma)=\sum_{i, j} n_{i, j}
$$

that $\chi(\Omega)=1-\operatorname{rk}(F)$, and that $\chi(\Gamma)=\sum_{i}\left(1-\operatorname{rk}\left(H_{i}\right)\right)$.

\section{One-relator pushouts}

We consider a branched map $f$ from a combinatorial complex $Y$ to a one-relator complex $X$ - that is, $X$ is a combinatorial complex with a single 2-cell. Suppose $w: S \rightarrow \Omega$ is the attaching map defining $X$, and the map $\lambda: P \rightarrow \Gamma$ defines $Y$. In this section we will see that the realization of the pushout $\Gamma_{W}$ of $\Gamma$ and $S$ along $P$ is the one-skeleton of a "best" one-relator complex $\hat{Y}$ that the map $Y \rightarrow X$ factors through. The dependence theorem implies that when $Y$ cannot be simplified in an obvious way, i.e. when $Y$ doesn't have any free faces, then $\chi(Y) \leq \chi(\hat{Y})$.

The components $P_{i}$ of $P$ are the boundaries of the 2-cells of $Y$. The degree of branching of $P_{i}$ under $f$ is denoted by $n_{i}$, and is the degree of the covering map $\left.\sigma\right|_{P_{i}}: P_{i} \rightarrow S$. Clearly

$$
\sum_{i}\left(n_{i}-1\right)=\operatorname{deg}(\sigma)-\#\{e \mid e \text { is a two-cell in } Y\} .
$$


Definition 3.1 (One-relator pushout). The one-relator complex defined by the map $w: S \rightarrow \Gamma_{W}$ is denoted by $\hat{Y}$, and is called the one-relator pushout of $Y$. The map $l$ extends to a branched map $\hat{Y}$ to $X$ (also denoted by $l$ ). By Remark 2.13. $\hat{Y}$ has the following universal property:

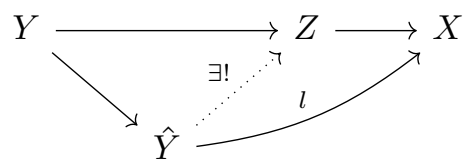

whenever $Z \rightarrow X$ is a morphism of degree one.

Similarly, the immersed one-relator pushout $\hat{Y}^{I}$ is the complex defined by the natural map $S \rightarrow \Gamma_{W}^{I}$. It enjoys a similar universal property for immersions $Z \rightarrow X$ of degree one.

In the context of one-relator complexes, the dependence theorem gives a relation between the Euler characteristics of $Y$ and $\hat{Y}$.

Corollary 3.2 (One-relator pushout inequality). Let $f: Y \rightarrow X$ be a branched map from a compact combinatorial complex $Y$ to a one-relator complex $X$ defined by $w: S \rightarrow \Omega$, with $w$ indivisible. If the restriction $\left.f\right|_{\partial Y}: \partial Y \rightarrow w\left(E_{S}\right)$ is not at least two-to-one then

$$
\chi(Y)+\sum_{i}\left(n_{i}-1\right) \leq \chi(\hat{Y}) \leq \chi\left(\hat{Y}^{I}\right) .
$$

In particular, if $Y$ has no free faces and $f$ is an immersion then $\chi(Y) \leq \chi(\hat{Y})$.

Proof. By (1),

$$
\chi(Y)+\sum_{i}\left(n_{i}-1\right)=\chi(\Gamma)+\operatorname{deg}(\sigma),
$$

so if $\chi(Y)+\sum_{i}\left(n_{i}-1\right)>\chi(\hat{Y})$ then $\chi(\Gamma)+\operatorname{deg}(\sigma)>\chi\left(\Gamma_{W}\right)+1$ and by the dependence theorem for each edge $e$ of of $\Gamma_{W},\left|\partial W \cap W_{e}\right| \geq 2$. The map $\partial W \rightarrow w\left(E_{S}\right)$ is therefore at least two-to one, and since $\partial Y=\partial \boldsymbol{W}$, so is $\left.f\right|_{\partial Y}$. This proves the first inequality.

The second inequality is clear since the one-skeleton $\Gamma_{W}^{I}$ of $\hat{Y}^{I}$ is obtained from the one-skeleton $\Gamma_{W}$ of $\hat{Y}$ by folding.

\section{Proof of the dependence theorem}

\subsection{Stackings}

As well as the adjunction space, the second tool that we will use is the notion of a stacking from [LW17. In that paper, a stacking of a map $\boldsymbol{w}: \boldsymbol{S} \rightarrow \boldsymbol{\Omega}$ was defined to be a lift of $w$ to an embedding into $\Omega \times \mathbb{R}$ (where $\mathbb{R}$ denotes the real numbers). Here, we use an equivalent, combinatorial, version of the definition. Given an injection of sets $\alpha: C \rightarrow D$ and a total order $\leq$ on $D$, we let $\alpha^{*}(\leq)$ denote the pullback order on $C$. 
Definition 4.1 (Stacking). Let $w: S \rightarrow \Omega$ be an immersion of graphs. A stacking of $w$ is a collection of orders $\leq_{x}$ on $w^{-1}(x)$ for $x \in w(S)$, such that $\alpha^{*}\left(\leq_{\alpha(e)}\right)=\leq_{e}$ for each $e \in w\left(E_{S}\right)$ and $\alpha=\iota$ or $\alpha=\tau$.

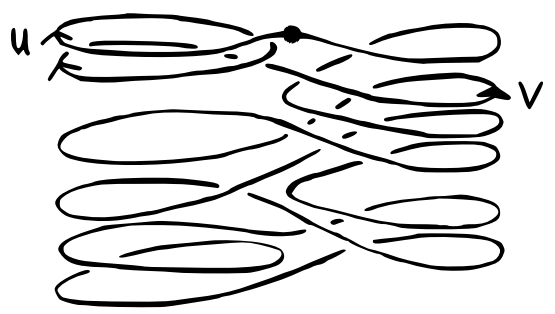

Figure 2: A stacking gives an inclusion $\widetilde{\boldsymbol{w}}: \boldsymbol{S} \hookrightarrow \boldsymbol{\Omega} \times \mathbb{R}$ and vice-versa. This is a picture of a stacking of (the realization of) the word $w=u u v u v v U U V U V V$ in the rose with two petals. This word can be written as a commutator in two inequivalent ways (see $[\mathrm{BF} 05]$ ).

Lemma 4.2 (Loo-roll lemma [LW17, Lemma 17]). Any indivisible immersion $w: S \rightarrow \Omega$ from a cycle to a graph has a stacking.

For the rest of the paper we will write realizations in normal rather than boldface font.

\subsection{Computing the characteristic of $W$}

In this subsection, we observe that Theorem 2.21 can be proved by estimating the Euler characteristic of a certain chain complex $\mathcal{C}$ naturally associated to any graph of graphs $W$. All coefficients are in a fixed but arbitrary field.

The chain complex $\mathcal{C}$ is

$$
0 \rightarrow H_{1}\left(W_{E}\right)=\bigoplus_{e \in E_{\Gamma_{W}}} H_{1}\left(W_{e}\right) \stackrel{\partial}{\rightarrow} H_{1}\left(W_{V}\right)=\bigoplus_{v \in V_{\Gamma_{W}}} H_{1}\left(W_{v}\right) \rightarrow 0,
$$

with boundary map $\partial=\tau_{\#}-\iota_{\#}$. As usual, the Euler characteristic of a chain complex is the alternating sum of the dimensions of its terms, so

$$
\chi(\mathcal{C})=b_{1}\left(W_{V}\right)-b_{1}\left(W_{E}\right) .
$$

Lemma 4.3. Let $W$ be any graph of graphs with connected vertex and edge graphs, and with underlying graph $\Gamma_{W}$. Then

$$
\chi(W)+\chi(\mathcal{C})=\chi\left(\Gamma_{W}\right) .
$$

Proof. By definition,

$$
\begin{aligned}
\chi(W) & =\chi\left(W_{V}\right)-\chi\left(W_{E}\right) \\
& =b_{0}\left(W_{V}\right)-b_{1}\left(W_{V}\right)-b_{0}\left(W_{E}\right)+b_{1}\left(W_{E}\right) \\
& =-\left(b_{1}\left(W_{V}\right)-b_{1}\left(W_{E}\right)\right)+\left(b_{0}\left(W_{V}\right)-b_{0}\left(W_{E}\right)\right) .
\end{aligned}
$$


Since the vertex and edge spaces are connected, $\chi\left(\Gamma_{W}\right)=b_{0}\left(W_{E}\right)-b_{0}\left(W_{V}\right)$, and the result follows.

When $W$ is the adjunction space associated to a branched map, we obtain that estimating $\chi(\mathcal{C})$ suffices to prove Theorem 2.21 .

Lemma 4.4. Let $h$ be a branched map of combinatorial complexes as above, and let $W$ be the horizontal graph of spaces for the adjunction space. Then

$$
\chi(\Gamma)+\chi(\mathcal{C})=\chi\left(\Gamma_{W}\right) .
$$

Proof. This is immediate from the previous lemma, because

$$
\chi(W)=\chi(\Gamma)+\chi(S)-\chi(P),
$$

but $S$ and $P$ are disjoint unions of circles, and so $\chi(S)=\chi(P)=0$.

\subsection{Fiberwise filtering $W$}

Let $W$ be the horizontal graph-of-graphs decomposition for the adjunction space of a branched map, and consider the chain complex $\mathcal{C}$ indexed by the graph $\Gamma_{W}$. In this section we use stackings to replace $\mathcal{C}$ by a pair of chain complexes $\mathcal{C}^{ \pm}$ indexed by $S$ and which have easily computable characteristic.

Let $W_{x}=\left(S_{x} \sqcup \Gamma_{x}, P_{x}, \lambda, \sigma\right)$ be a (bipartite) vertex or edge graph of $W$, where $S_{x}=W_{x} \cap S, \Gamma_{x}=W_{x} \cap \Gamma, P_{x}=W_{x} \cap P$. For each vertex $s \in S_{x}$, let $P_{s}=\sigma^{-1}(s)$.

Suppose that $w: S \rightarrow \Omega$ has a stacking, which we pull back to a stacking of $w: S \rightarrow \Gamma_{W}$. For $s \in S_{x}$ define

$$
W_{x}^{+}(s)=\Gamma_{x} \cup\left\{t \mid t \leq_{x} s\right\} \cup\left\{p \mid \sigma(p) \leq_{x} s\right\}
$$

and

$$
W_{x}^{-}(s)=\Gamma_{x} \cup\left\{t \mid s \leq_{x} t\right\} \cup\left\{p \mid s \leq_{x} \sigma(p)\right\} .
$$

Let $s+1$ be the successor of $s$ and $s-1$ be the predecessor of $s$, when defined, and interpret $W_{x}^{+}(s-1)$ as $\Gamma_{x}$ if $s$ is minimal and $W_{x}^{-}(s+1)$ as $\Gamma_{x}$ if $s$ is maximal. The order $\leq_{x}$ gives two filtrations of $W_{x}$ by the sublevel sets $W_{x}^{ \pm}(s)$.

$$
\Gamma_{x} \subsetneq \cdots \subsetneq W_{x}^{+}(s-1) \subsetneq W_{x}^{+}(s) \subsetneq W_{x}^{+}(s+1) \subsetneq \cdots \subsetneq W_{x}
$$

and

$$
\Gamma_{x} \subsetneq \cdots \subsetneq W_{x}^{-}(s+1) \subsetneq W_{x}^{-}(s) \subsetneq W_{x}^{-}(s-1) \subsetneq \cdots \subsetneq W_{x} .
$$

For $s \in S_{x}$, define

$$
A^{ \pm}(s)=H_{1}\left(W_{x}^{ \pm}(s)\right) / H_{1}\left(W_{x}^{ \pm}(s \mp 1)\right) .
$$


The quotient group $A^{ \pm}(s)$ represents the additional first homology gained when going from $W_{x}^{ \pm}(s \mp 1)$ to $W_{x}^{ \pm}(s)$. See Figure 4. Summing over $s \in S_{x}$, we have

$$
H_{1}\left(W_{x}\right) \cong \bigoplus_{s \in S_{x}} A^{ \pm}(s) .
$$

The attaching map $\alpha_{e}: W_{e} \rightarrow W_{\alpha(e)}$ is injective on $S$-vertices and respects the orders $\leq_{*}$, so $\alpha^{*}\left(\leq_{\alpha(e)}\right)=\leq_{e}$, and there are therefore restrictions

$$
W_{e}^{ \pm}(s) \rightarrow W_{\alpha(e)}^{ \pm}(\alpha(s))
$$

such that

$$
\alpha_{e}\left(W_{e}^{ \pm}(s \mp 1)\right) \subseteq W_{\alpha(e)}^{ \pm}(\alpha(s \mp 1)) \subseteq W_{\alpha(e)}^{ \pm}(\alpha(s) \mp 1) .
$$

Because $h$ is a branched map, by Lemma 2.5 each $\alpha_{e}: P_{e} \rightarrow P_{\alpha(e)}$ is injective, so $\alpha_{s}: P_{s} \rightarrow P_{\alpha(s)}$ is as well, so there are induced injections

$$
\alpha_{s, \#}^{ \pm}: A^{ \pm}(s) \hookrightarrow A^{ \pm}(\alpha(s)) .
$$

Again, summing over $s \in S_{x}$, there are maps

$$
\alpha_{e, \#}^{ \pm}=\bigoplus_{s \in S_{e}} \alpha_{s, \#}^{ \pm}: \bigoplus_{s \in S_{e}} A^{ \pm}(s) \hookrightarrow \bigoplus_{s \in S_{\alpha(e)}} A^{ \pm}(s) .
$$

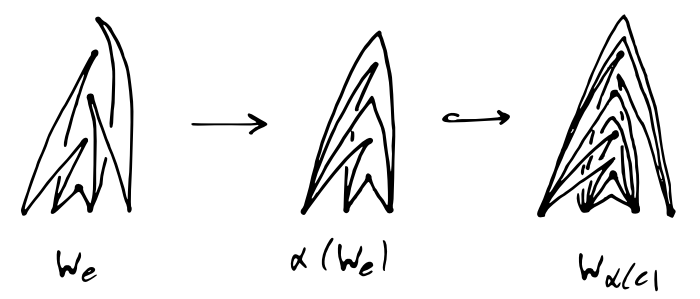

Figure 3: The map $\alpha: W_{e} \rightarrow W_{\alpha(e)}$ is injective on $P_{e}$ and induces an injection $A^{ \pm}(s) \hookrightarrow A^{ \pm}(\alpha(s))$. In this example two vertices of $\Gamma_{e}$ are identified in $\Gamma_{\alpha(e)}$. The map $\alpha$ respects the sublevelset filtrations (2) and (3). Here we have drawn $S_{x}$ as sitting "above" the $\Gamma_{x}$ so this picture should be thought of as illustrating the filtration (2).

We now define a pair of auxiliary chain complexes $\mathcal{C}^{ \pm}$by replacing each $H_{1}\left(W_{x}\right)$ in $\mathcal{C}$ using the isomorphism (4), using the sum of the maps from (6) as the boundary map, that is

$$
\partial^{ \pm}:=\bigoplus_{e \in E_{\Gamma_{W}}} \tau_{e, \#}^{ \pm}-\iota_{e, \#}^{ \pm} .
$$


And so

$$
\mathcal{C}^{ \pm}=\left(0 \rightarrow \bigoplus_{e \in E_{\Gamma_{W}}} \bigoplus_{s \in S_{e}} A^{ \pm}(s) \stackrel{\partial^{ \pm}}{\longrightarrow} \bigoplus_{v \in V_{\Gamma_{W}}} \bigoplus_{s \in S_{v}} A^{ \pm}(s) \rightarrow 0\right)
$$

By $(4), \chi\left(\mathcal{C}^{ \pm}\right)=\chi(\mathcal{C})$. Since

$$
V_{S}=\bigsqcup_{v \in V_{\Gamma_{W}}} S_{v} \text { and } E_{S}=\bigsqcup_{e \in E_{\Gamma_{W}}} S_{e}
$$

after reindexing, 7] becomes

$$
\mathcal{C}^{ \pm}=\left(0 \rightarrow \bigoplus_{e \in E_{S}} A^{ \pm}(e) \rightarrow \bigoplus_{v \in V_{S}} A^{ \pm}(v) \rightarrow 0\right)
$$

with boundary maps coming from (5).

These auxiliary chain complexes enable us to relate $\chi(\mathcal{C})$ to the vector spaces $A^{ \pm}(s)$ that come from the filtrations of the $W_{x}$.

Lemma 4.5. Suppose $S$ is a cycle. Then

$$
\max \left\{\operatorname{dim}\left(A^{ \pm}(s)\right) \mid s \in S\right\} \leq \chi(\mathcal{C}) .
$$

The proof uses the following naive estimate.

Remark 4.6. Let $a_{1}, \ldots, a_{n}$ and $b_{1}, \ldots, b_{n-1}$ be non-negative integers, and suppose that $a_{i} \geq b_{i} \leq a_{i+1}$ for $i=1 \ldots n-1$. Then

$$
a_{1}-b_{1}+\cdots-b_{n-1}+a_{n} \geq \max \left\{a_{i}, b_{i}\right\} .
$$

Proof of Lemma 4.5. Pick an edge $g \in w\left(E_{S}\right) \subseteq E_{\Gamma_{W}}$, and let $m^{+}$and $m^{-}$be the minimal and maximal elements of $S_{g}$ with respect to the order $\leq_{g}$. Since $m^{ \pm}$is minimal/maximal,

$$
V_{W_{g}^{ \pm}\left(m^{ \pm}\right)}=\Gamma_{g} \cup\left\{m^{ \pm}\right\}
$$

and

$$
E_{W_{g}^{ \pm}\left(m^{ \pm}\right)}=\left\{p \mid \sigma(p)=m^{ \pm}\right\} .
$$

By Lemma $2.19 W_{g}$ is simple, so if $p \in E_{W_{g}^{ \pm}\left(m^{ \pm}\right)}$then $p$ is determined by $\lambda(p)$, and $W_{g}^{ \pm}\left(m^{ \pm}\right)$is therefore $\Gamma_{g}$ with $\lambda\left(P_{m^{ \pm}}\right)$coned off, so $A^{+}\left(m^{+}\right) \cong A^{-}\left(m^{-}\right) \cong$ 0 . Removing $m^{ \pm}$from $S$ therefore doesn't change the characteristic of the chain complexes $\mathcal{C}^{ \pm}$, i.e.

$$
\chi\left(\mathcal{C}^{ \pm}\right)=\chi\left(\left.\mathcal{C}^{ \pm}\right|_{S \backslash m^{ \pm}}\right)
$$

where

$$
\left.\mathcal{C}^{ \pm}\right|_{S \backslash m^{ \pm}}=\left(0 \rightarrow \bigoplus_{e \in E_{S} \backslash m^{ \pm}} A^{ \pm}(e) \rightarrow \bigoplus_{v \in V_{S}} A^{ \pm}(v) \rightarrow 0\right) .
$$


The chain complex $\left.\mathcal{C}^{ \pm}\right|_{S \backslash m^{ \pm}}$is over an interval $S \backslash m^{ \pm}$, which makes its Euler characteristic easy to estimate. Label and reorient $S$ so that $V_{S}=\left\{v_{1}^{ \pm}, \ldots, v_{n}^{ \pm}\right\}$ and $E_{S}=\left\{m^{ \pm}, e_{1}^{ \pm}, \ldots, e_{n-1}^{ \pm}\right\}$with $\iota\left(e_{i}^{ \pm}\right)=v_{i}^{ \pm}($for $i=1, \ldots, n)$ and $\tau\left(e_{i}^{ \pm}\right)=$ $v_{i+1}^{ \pm}($for $i=1, \ldots, n-1)$. Set $a_{i}^{ \pm}=\operatorname{dim}\left(A^{ \pm}\left(v_{i}^{ \pm}\right)\right)$and $b_{j}^{ \pm}=\operatorname{dim}\left(A^{ \pm}\left(e_{j}^{ \pm}\right)\right)$. Then

$$
\chi(\mathcal{C})=\chi\left(\mathcal{C}^{ \pm}\right)=a_{1}^{ \pm}-b_{1}^{ \pm}+a_{2}^{ \pm}-\cdots+a_{n-1}^{ \pm}-b_{n-1}^{ \pm}+a_{n}^{ \pm} .
$$

Since $\alpha: A^{ \pm}(e) \rightarrow A^{ \pm}(\alpha(e))$ is injective, $a_{i}^{ \pm} \geq b_{i}^{ \pm} \leq a_{i+1}^{ \pm}$for $i=1, \ldots, n-1$, and

$$
\chi(\mathcal{C}) \geq \max \left\{a_{i}^{ \pm}, b_{i}^{ \pm}\right\}=\max \left\{\operatorname{dim}\left(A^{ \pm}(s)\right) \mid s \in S\right\} \geq 0
$$

by Remark 4.6 .

Remark 4.7. It is not clear from the start that $\chi(\mathcal{C})$ is non-negative. It follows from Mayer-Vietoris that the chain complexes $\mathcal{C}^{ \pm} \backslash m^{ \pm}$, and therefore $\mathcal{C}^{ \pm}$, have their homology concentrated in dimension 0 .

$$
\chi\left(\mathcal{C}^{ \pm}\right)=\operatorname{dim}\left(H_{0}\left(\mathcal{C}^{ \pm}\right)\right)
$$

The special case $\chi(\Gamma)=\chi\left(\Gamma_{W}\right)$ is of some interest since it implies the theorems of Baumslag and Stallings. In these cases $\chi(\mathcal{C})=0$, and by Lemma 4.5 $\operatorname{dim}\left(A^{ \pm}(s)\right)=0$ for all $s \in S$. By (4), $H_{1}\left(W_{x}\right)=0$ for all $x \in \Gamma_{W}$, but a connected graph with trivial homology is a tree. If $\operatorname{deg}(\sigma) \geq 2$ then no $s \in S_{x}$ has valence one, so there are at least two valence-one vertices in $\Gamma_{x}$, hence $\lambda$ is strongly reducible, and therefore reducible. This case is argued differently in the paper [Lou13. There it was shown directly that the vertices in $\Gamma_{x}$ are cutpoints in $W_{x}$, and acylindricity of the associated graph of groups $\Delta$ then implied that the edge and vertex spaces are trees. Since this is not true in general, we use stackings to argue indirectly that if $\chi(\mathcal{C})<\operatorname{deg}(\sigma)-1$ then the edge spaces have "treelike" features, and ultimately, valence one vertices.

\subsection{The up-down lemma and the proof of Theorem 2.21}

The final ingredient of the proof of the dependence theorem is the up-down lemma. To formulate it, we first recapitulate some of the discussion from Section 4.3 in general terms.

Consider a finite bipartite graph $B=(U \sqcup V, E, \sigma, \lambda)$ with an order $\leq$ on $V$. For $v \in V$ define

$$
B^{+}(v)=U \cup\left\{v^{\prime} \mid v^{\prime} \leq v\right\} \cup\{e \mid \sigma(e) \leq v\}
$$

and

$$
B^{-}(v)=U \cup\left\{v^{\prime} \mid v^{\prime} \geq v\right\} \cup\{e \mid \sigma(e) \geq v\} .
$$

Let

$$
A^{ \pm}(v)=H_{1}\left(B^{ \pm}(v)\right) / H_{1}\left(B^{ \pm}(v \mp 1)\right),
$$

where we interpret $B^{+}(v-1)$ as $U$ if $v$ is minimal and $B^{-}(v+1)$ as $U$ if $v$ is maximal. A vertex $v \in V$ is good if

$$
\max \left\{\operatorname{dim}\left(A^{ \pm}(v)\right)\right\}=\operatorname{val}(v)-1 .
$$


A vertex $u \in U$ is good if it has valence one.

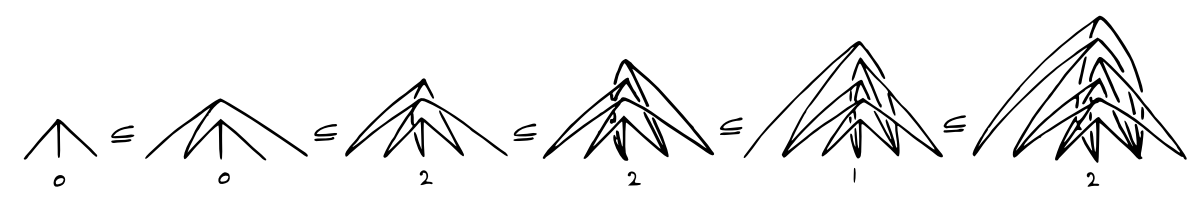

Figure 4: Illustration of a filtration associated to an order $\leq$ on a (simple) bipartite graph $B$. The elements of $U$ are all drawn at the same level, and elements of $V$ are placed vertically. To keep the pictures uncluttered we omit elements of $U$ which aren't connected to vertices in $V \cap B^{+}(v)$. The number below each graph is the dimension of $A^{+}(v)$ for the vertex $v$ added at that stage. The graph $B$ has $6+6$ vertices and 18 edges, for a characteristic of -6 , and is connected with first betti number $0+0+2+2+1+2$.

Lemma 4.8 (Up-down lemma). Let $B$ be a simple connected bipartite graph which is not a point. Let $\leq$ be an order on $V$. Then

$$
\mid\{p \in V \cup U \mid p \text { is good. }\} \mid \geq 2 .
$$

Proof. The proof is by induction on $|V|$. Suppose that $|V|=1$. If $|U|=1$ then $V=\{v\}, U=\{u\}, v$ has valence $1, \operatorname{dim}\left(A^{ \pm}(v)\right)=\operatorname{val}(v)-1=0$, so $v$ is good, and $\left|\lambda^{-1}(u)\right|=1$ so $u$ has valence one, so is good. If $|U| \geq 2$ then there are $|U| \geq 2$ valence one vertices in $U$.

Suppose that $|V| \geq 2$, and let $m^{-}$and $m^{+}$be the maximal and minimal elements of $V$, respectively. If $m^{-}$and $m^{+}$are both good then we are done.

The long exact sequence for the pair $\left(B, B^{+}\left(m^{-}-1\right)\right)$ reduces to the exact sequence

$$
0 \rightarrow A^{+}\left(m^{-}\right) \rightarrow H_{1}\left(B, B^{+}\left(m^{-}-1\right)\right) \rightarrow H_{0}\left(B^{+}\left(m^{-}-1\right)\right) \rightarrow H_{0}(B) \rightarrow 0 .
$$

Since $B \backslash B^{+}\left(m^{-}-1\right)$ has one vertex $m^{-}$and has val $\left(m^{-}\right)$edges connecting $B^{+}\left(m^{-}-1\right)$ to $m^{-}$, the relative homology group $H_{1}\left(B, B^{+}\left(m^{-}-1\right)\right)$ is $\operatorname{val}\left(m^{-}\right)-1$ dimensional. Since $B$ is connected, $\operatorname{dim}\left(H_{0}(B)\right)=1$. Suppose now that $m^{-}$is not good. Since $B$ is simple, $\operatorname{dim}\left(A^{-}\left(m^{-}\right)\right)=0$, and since $m^{-}$is not $\operatorname{good}, \operatorname{dim}\left(A^{+}\left(m^{-}\right)\right)<\operatorname{val}\left(m^{-}\right)-1$, so by $(8) \operatorname{dim}\left(H_{0}\left(B^{+}\left(m^{-}-1\right)\right)\right)>1$, and $B^{+}\left(m^{-}-1\right)$ is therefore not connected and $B \backslash m^{-}$has at least two connected components. Let $B_{m^{-}}$be the closure of a connected component of $B \backslash m^{-}$ which doesn't contain $m^{+}$. By induction on $|V|, B_{m^{-}}$has at least two good vertices, one of which is not $m^{-}$. Let $g$ be this vertex. If $m^{+}$is good then $m^{+}$ and $g$ are both good. Argue symmetrically if $m^{-}$is good and $m^{+}$is not good.

Thus we assume both $m^{-}$and $m^{+}$are not good. Again, let $B_{m^{-}}$be the closure of a connected component of $B \backslash m^{-}$which doesn't contain $m^{+}$, and let $B_{m^{+}}$be the closure of a connected component of $B \backslash m^{+}$which doesn't contain $m^{-}$. The vertices $m^{-}$and $m^{+}$are good in $B_{m^{-}}$and $B_{m^{+}}$, respectively, and 
$B_{m^{-}}$and $B_{m^{+}}$are disjoint. By induction on $|V|, B_{m^{-}}$and $B_{m^{+}}$each contain at least two good vertices, at least one of which is not $m^{-}$or $m^{+}$, respectively. A good vertex in $B_{m^{-}}$which is not $m^{-}$is good in $B$, and a good vertex in $B_{m^{+}}$ which is not $m^{+}$is good in $B$ as well, so $B$ has at least two good vertices.

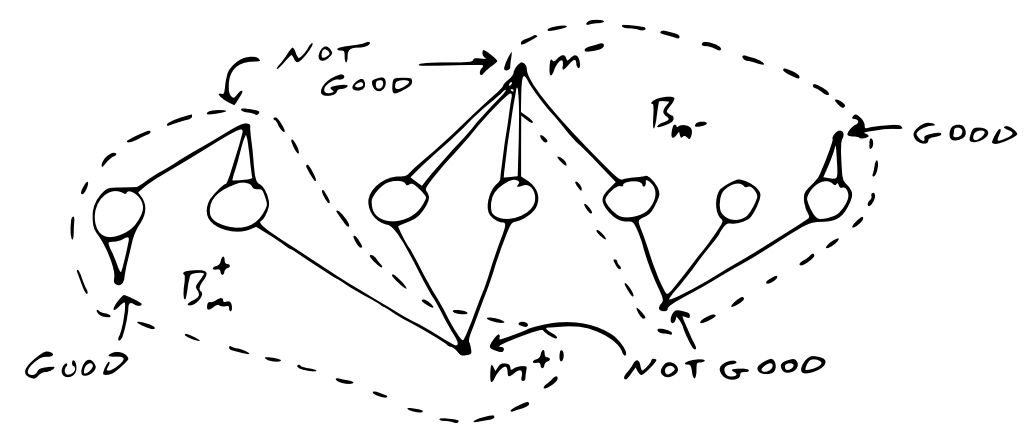

Figure 5: Illustration for Lemma 4.8. In this case neither $m^{-}$nor $m^{+}$is good. We picture $U$ as sitting below $m^{-}$and above $m^{+}$.

With the up-down lemma in hand, we can finally prove the dependence theorem.

Proof of Theorem 2.21. We prove the contrapositive. Suppose that

$$
\chi(\Gamma)+\operatorname{deg}(\sigma)-1>\chi\left(\Gamma_{W}\right) .
$$

Our goal is to prove that $W$ is strongly independent.

By Lemma 4.5 $\chi(\mathcal{C})$ is bounded from below by

$$
\max _{x \in \Gamma_{W}} \max _{s \in S_{x}}\left\{\operatorname{dim}\left(A^{ \pm}(s)\right)\right\}
$$

so

$$
\chi(\Gamma)+\max _{x \in \Gamma_{W}} \max _{s \in S_{x}}\left\{\operatorname{dim}\left(A^{ \pm}(s)\right)\right\} \leq \chi(\Gamma)+\chi(\mathcal{C})=\chi\left(\Gamma_{W}\right),
$$

where the last equality is given by Lemma 4.4. If $\chi(\Gamma)+\operatorname{deg}(\sigma)-1>\chi\left(\Gamma_{W}\right)$ then

$$
\max _{x \in \Gamma_{W}} \max _{s \in S_{x}}\left\{\operatorname{dim}\left(A^{ \pm}(s)\right)\right\}<\operatorname{deg}(\sigma)-1 .
$$

To show that $W$ is strongly independent, we need to show that $\left|\partial W \cap W_{e}\right| \geq 2$ for each $e \in w\left(E_{S}\right) \subseteq E_{\Gamma_{W}}$.

To that end, choose $e \in w\left(E_{S}\right)$. By Lemma 2.19(i), $W_{e}$ is a simple graph, so we may apply the up-down lemma to $W_{e}$ by setting $B=W_{e}, V=S_{e}, U=\Gamma_{e}$, $E=P_{e}$, and $\leq=\leq_{e}$. Remark 2.20 asserts that $\operatorname{deg}(\sigma)=\operatorname{val}(s)$, so (9) implies that $\operatorname{dim}\left(A^{ \pm}(s)\right)<\operatorname{val}(s)-1$ for all $s \in S_{e}$. In particular, no vertex in $S_{e}$ is good. Since the up-down lemma guarantees two good vertices in $W_{e}$, it follows 
that there are two good vertices in $\Gamma_{e}$. A good vertex in $\Gamma_{e}$ has valence one, so $\left|\partial W \cap W_{e}\right| \geq 2$.

This is true for all $e \in w\left(E_{S}\right)$, but this is precisely what it means for the map $\lambda: P \rightarrow \Gamma$ to be strongly independent.

\section{Stallings; Magnus and Lyndon; Duncan-Howie}

In this section we show how the dependence theorem implies its predecessors mentioned in the introduction. We have already seen that it implies Theorem 1.16, which in turn implies Baumslag's theorem. We next state a generalization of Stallings' theorem and explain how it follows as well. In the following subsection we explain how the dependence theorem implies Magnus' Freiheitssatz and Lyndon's asphericity theorem. Finally, we explain how the dependence theorem implies a strengthening of the theorem of Duncan-Howie.

\subsection{Conjugacy and homology}

A homomorphism of free groups $f: H \rightarrow F$ induces a map $f_{\sim}: H / \sim \rightarrow F / \sim$ on sets of conjugacy classes. A 1983 theorem of Stallings, which we also think of as a kind of dependence theorem, relates $f_{\sim}$ to the induced map on abelianizations, $f_{\#}: H_{1}(H) \rightarrow H_{1}(F)$ [Sta83a, Theorem 5.3].

Theorem (Stallings). Let $f: H \rightarrow F$ be an injection of finitely generated free groups. If $f_{\#}$ is injective then so is $f_{\sim}$.

(A homomorphism $f$ for which $f_{\sim}$ is injective is sometimes called a Frattini embedding; cf. OS04.)

In this section we quantify Stallings' theorem, and compare how badly $f_{\sim}$ and $f_{\#}$ may fail to be injective. In the case of $f_{\#}$, the failure of injectivity is measured by the rank of the kernel. To measure the failure of $f_{\sim}$ to be injective, we define

$$
\gamma(f)=\max _{[v] \in F / \sim}\left\{\left|f_{\sim}^{-1}([v])\right|\right\} \in \mathbb{N} \cup\{\infty\},
$$

the maximal number of conjugacy classes in $H$ that are identified in $F$. Using this terminology, Stallings' theorem asserts: if $\gamma(f)>1$ then $\operatorname{rk}\left(\operatorname{ker}\left(f_{\#}\right)\right)>0$.

The main result of this section is a corollary of the dependence theorem that strengthens Stallings' theorem by comparing $\operatorname{rk}\left(\operatorname{ker}\left(f_{\#}\right)\right)$ to $\gamma(f)$.

Corollary 5.1. Let $f: H \rightarrow F$ be an injection of finitely generated free groups. Then

$$
\operatorname{rk}\left(\operatorname{ker}\left(f_{\#}\right)\right) \geq \gamma(f)-1 \text {. }
$$

Proof. By Marshall Hall's theorem, $f$ identifies $H$ with a free factor in some subgroup $K$ of finite index in $F$. Each conjugacy class of $F$ splits into at most $|F: K|$ conjugacy classes in $K$, and does not split any further in $f(H)$. Therefore, $\gamma(f) \leq|F: K|$, and in particular is finite. 
The proof proceeds by induction on $m=\gamma(f)$. In the base case, $m=1$, there is nothing to prove, so we assume that $m \geq 2$. We may also assume that $H$ and $F$ are finitely generated. Let $u_{1}, \ldots, u_{m}$ be a collection of non-conjugate elements realizing $\gamma(f)$.

Since free groups have unique roots, for each $u_{j}$ there is a unique $v_{j} \in H$ such that $u_{j}=v_{j}^{k_{j}}$, with $k_{j} \geq 1$ maximal. Using uniqueness of roots again, it follows that $\left\{\left\langle v_{j}\right\rangle\right\}$ forms a malnormal family of cyclic subgroups of $H$. Since the $f\left(u_{j}\right)$ are all conjugate to each other, the $f\left(v_{j}\right)$ are all conjugate into some common cyclic subgroup $\langle w\rangle$ of $F$. Therefore, each $f\left(v_{j}\right)$ is conjugate to $w^{n_{j}}$ for some unique integer $n_{j}$. As in the introduction, these data define a graph of groups $\Delta$ and $f$ extends to a homomorphism $\phi: \pi_{1}(\Delta) \rightarrow F$. Let $L=\phi\left(\pi_{1}(\Delta)\right) \leq F$.

Since $f(H)$ is contained in $L$ we have $\operatorname{rk}\left(\operatorname{im}\left(f_{\#}\right)\right) \leq \operatorname{rk}(L)$ and so the ranknullity lemma applied to $f_{\#}$ gives

$$
\operatorname{rk}\left(\operatorname{ker}\left(f_{\#}\right)\right)=\operatorname{rk}(H)-\operatorname{rk}\left(\operatorname{im}\left(f_{\#}\right)\right) \geq \operatorname{rk}(H)-\operatorname{rk}(L) .
$$

If the malnormal family $\left\{\left\langle v_{j}\right\rangle\right\}$ is dependent then Theorem 1.16 implies that

$$
\operatorname{rk}(H)-\operatorname{rk}(L) \geq\left(\sum_{i=1}^{m} n_{i}\right)-1 \geq m-1 .
$$

These two estimates together imply the result, so it remains to deal with the case in which $\left\{\left\langle v_{j}\right\rangle\right\}$ is independent.

After permuting indices and conjugating the $v_{j}$ appropriately, this means that

$$
H=K *\left\langle v_{m}\right\rangle
$$

and $v_{j} \in K$ for $j<m$. Therefore, by the inductive hypothesis applied to $\left.f\right|_{K}: K \rightarrow F$, we have $\operatorname{rk}\left(\operatorname{ker}\left(\left.f_{\#}\right|_{H_{1}(K)}\right)\right) \geq m-2$. Since $f\left(v_{1}\right)^{n_{m}}$ is conjugate to $f\left(v_{m}\right)^{n_{1}}$, the class

$$
c=n_{m}\left[v_{1}\right]-n_{1}\left[v_{m}\right]
$$

is non-zero in $H_{1}(H)$, is contained in the kernel of $f_{\#}$, but is not in $H_{1}(K)$. Therefore,

$$
\operatorname{rk}\left(\operatorname{ker}\left(f_{\#}\right)\right) \geq \operatorname{rk}\left(\operatorname{ker}\left(\left.f_{\#}\right|_{H_{1}(K)}\right)\right)+1 \geq m-1
$$

as required.

Remark 5.2. Corollary 5.1 is sharp. Let $F=\langle a, b\rangle$ and

$$
H=\left\langle a, b a b^{-1}, \ldots, b^{n-2} a b^{2-n}, b^{n-1} a b^{1-n}\right\rangle
$$

with $f$ the inclusion map. The $n$ basis elements $b^{i} a b^{-i}$ of $H$ are conjugate in $F$ and $\operatorname{rk}\left(\operatorname{ker}\left(f_{\#}\right)\right)=n-1$. 


\subsection{The Freiheitssatz and Lyndon asphericity}

We again consider a one-relator group $G=F /\langle\langle w\rangle$. As usual, we think of $F$ as the fundamental group of a graph $\Omega$, and realise $w$ as an immersion $S \rightarrow \Omega$, where $S$ is a cycle. Note that $w$ may be a proper power $v^{k}$, where $k \geq 1$ is assumed to be maximal. In this section we show how Corollary 3.2 implies the Freiheitssatz and Lyndon asphericity. In what follows $X$ is the presentation complex $(\Omega, S, w)$ of the one-relator group $G$, where $w: S \rightarrow \Omega$ is the attaching map of the two cell, and $Z$ is the presentation complex of the one-relator group $(\Omega, S, v)$. There is a natural map $q: X \rightarrow Z$, equal to the identity on $\Omega$ and a $k$ sheeted cover on $S$. Note that $q$ is not a branched map in the sense of Definition 2.2 if $k>1$.

Definition 5.3 (Surface diagram). A singular surface diagram in $X$ is a morphism $f: Y \rightarrow X$ of combinatorial complexes, such that the link of every vertex in $Y$ is a union of points, cycles and intervals. A singular surface diagram $f: Y \rightarrow X$ is reduced if the induced map $q \circ f$ is a branched map.

This definition agrees with the usual notions of reduced disk and sphere diagram. The following theorem, which is the main theorem of this section, is a common generalization of Magnus' Freiheitssatz and Lyndon asphericity.

Theorem 5.4 (Magnus, Lyndon). Let $X$ be the presentation complex of a onerelator group, and $f: Y \rightarrow X$ a reduced singular surface diagram. If $\chi(Y) \geq 1$ then $w(S) \subseteq f(\partial Y)$.

Proof. If $w(S) \nsubseteq f(\partial Y)$ then certainly $w(S) \nsubseteq \subseteq q(f(\partial Y))$, so we may replace $X$ by $Z$. Let $\hat{Y}$ be the one-relator pushout of the map $Y \rightarrow Z$. By Corollary 3.2 . $\chi(Y) \leq \chi(\hat{Y})$, so $\chi(\hat{Y}) \geq 1$. Since $\hat{Y}$ is one-relator, and $v$ is indivisible, $\hat{Y}$ is the disk $D$, and $\Gamma_{W}=\partial D$ is a cycle. Since $Y \rightarrow Z$ is a branched map, it doesn't fold faces, but since $Y \rightarrow Z$ factors through $D$, no two two-cells in $Y$ share an edge. Thus $Y$ is a tree of disks. In this case, $\partial Y$ clearly surjects $w(S)$.

Magnus' Freiheitssatz Mag30, corresponding to the case when $Y$ is a disk, and Lyndon asphericity Lyn50, Coc54, corresponding to the case where $Y$ is a sphere, follow immediately.

Corollary 5.5 (Magnus' Freiheitssatz). Consider a one-relator group $G=$ $F /\langle\langle w\rangle$. If $H$ is a proper free factor of free group $F$ and the natural map $H \rightarrow G$ is not injective then $w$ is conjugate into $H$.

Proof. Let $H$ be a free factor of $F$ and $\gamma \in H$ an element that dies in $G$. Take $X$ to be a presentation complex of $G$ in such a way that $H$ is realized by a subgraph of the one-skeleton. We can realize $\gamma$ as an immersed loop in $X$ with image in the subgraph that carries $H$. Since $\gamma$ dies in $G, \gamma$ factors through a reduced disk diagram $f: Y \rightarrow X$ by van Kampen's lemma, in such a way that $\gamma$ surjects the boundary $\partial Y$. By Theorem 5.4 the restriction of $f$ to $\partial Y$ surjects $w(S)$, and hence $\gamma$ does too. Since $\gamma \in H$, it follows that $w$ is conjugate into $H$. 
Corollary 5.6 (Lyndon asphericity). Let $X$ be the presentation complex of a one-relator group $G=F /\langle w\rangle\rangle$. If $Y$ is homeomorphic to a 2-sphere, no combinatorial map $Y \rightarrow X$ is reduced.

\subsection{Roots of products of commutators}

Definition 5.7. Let $F$ be a free group. The genus or commutator length of an element $v \in F$ is defined to be the minimal $g \in \mathbb{N}$ such that

$$
v=\left[a_{1}, b_{1}\right] \cdots\left[a_{g}, b_{g}\right] .
$$

The Duncan-Howie theorem is an estimate on the commutator length of a proper power $v=w^{n}$ : it asserts that $n \leq 2 g-1$ [DH91. Here, we view it as a dependence theorem about maps $H \rightarrow F$ where $H$ is the fundamental group of a surface $\Sigma$ with boundary, and $\partial \Sigma$ maps to powers of conjugates of $w$. In this section, we prove another corollary of Theorem 2.21. which strengthens the Duncan-Howie theorem.

Corollary 5.8. Let $F$ be a free group and consider $v$ a non-trivial element which is both a $k$-th power and a product of $g$ commutators, that is there are $a_{i}, b_{i}, w \in F$ with $1 \leq i \leq g$ and

$$
v=\left[a_{1}, b_{1}\right] \cdots\left[a_{g}, b_{g}\right]=w^{k} .
$$

Then

$$
\operatorname{rk}\left(\left\langle a_{1}, \ldots, a_{g}, b_{1}, \ldots, b_{g}, w\right\rangle\right)+k-1 \leq 2 g .
$$

Since $w \neq 1$, the group $\left\langle a_{1}, \ldots, a_{g}, b_{1}, \ldots, b_{g}, w\right\rangle$ is a non-abelian free group, and hence has rank at least 2 . Therefore, $k \leq 2 g-1$, recovering the DuncanHowie estimate.

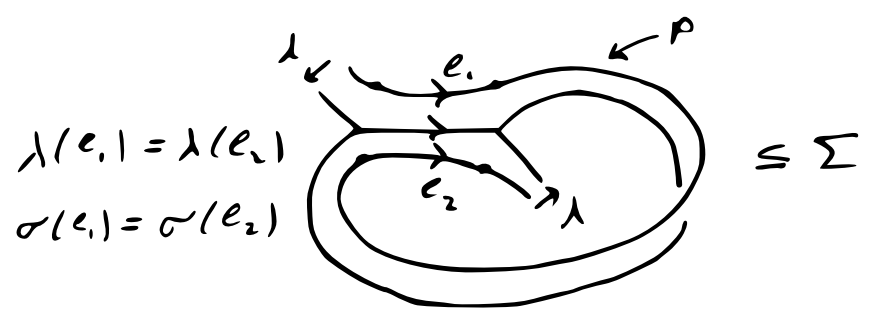

Figure 6: When $\Sigma$ is orientable the map $\lambda: P \rightarrow \Gamma$ is a branched map since otherwise $\Sigma$ contains a Möbius band.

Proof of Corollary 5.8. Represent the subgroup $\left\langle a_{i}, b_{i}\right\rangle \leq F$ by a map $f: \Sigma \rightarrow$ $\Omega$ from an orientable surface of genus $g$ with one boundary component, so that $\left.f\right|_{\partial \Sigma}$ represents the element $v$. We may assume that $f$ doesn't pinch any 
simply closed curves, and that $w$ is indivisible in $\left\langle a_{1}, \ldots, a_{g}, b_{1}, \ldots, b_{g}, w\right\rangle$. By Cul81, we may realize $\Sigma$ as the mapping cylinder of $\lambda: P \rightarrow \Gamma$, where $P$ is a cycle representing the boundary of $\Sigma$, with a morphism of graphs $h: \Gamma \rightarrow$ $\Omega$ representing $\left\langle a_{1}, \ldots, a_{g}, b_{1}, \ldots, b_{g}\right\rangle$. Orientability of $\Sigma$ implies that $\lambda$ is a branched map. See Figure 6. The induced map from the pushout $\Gamma_{W}$ surjects $\left\langle a_{1}, \ldots, a_{g}, b_{1}, \ldots, b_{g}, w\right\rangle$, and the inequality then follows from the dependence theorem.

\section{Subgroups of one-relator groups}

The results of this section show how $\pi(w)$ controls the subgroup structure of the one-relator group $G=F /\langle w\rangle\rangle$.

\subsection{Primitivity rank and $w$-subgroups}

Recall the definition of the primitivity rank $\pi(w)$ from the introduction (Definition 1.2. . We start with a few simple observations.

(i) The word $w$ is primitive in $F$ if and only if $\pi(w)=\infty$.

(ii) Unless $w$ is primitive, $\operatorname{rk}(F)$ is an upper bound for $\pi(w)$.

(iii) The word $w$ is a proper power if and only if $\pi(w)=1$.

(iv) If $w$ is contained in a subgroup $H \leq F$ and $w$ is primitive in $F$ then $w$ is primitive in $H$. Therefore, the primitivity rank of $w$ when viewed as an element of $H$ is at least the primitivity rank of $w$ when viewed as an element of $F$.

We now turn to the second definition needed for the main lemma.

Definition 6.1. Let $F$ be a free group and $w \in F$ a non-trivial element. A subgroup $K$ of $F$ is a $w$-subgroup if:

(i) $K$ contains $w$ as an imprimitive element;

(ii) $\operatorname{rk}(K)=\pi(w)$; and

(iii) every proper overgroup $K^{\prime}$ of $K$ in $F$ has $\operatorname{rk}\left(K^{\prime}\right)>\operatorname{rk}(K)$.

In the easiest case $w$-subgroups are cyclic; this occurs if and only if $\pi(w)=1$, i.e. when $w$ is a proper power $u^{k}$.

Example 6.2. If $w=u^{k} \in F$ with $k>1$ and $u$ not a proper power then $\langle u\rangle$ is the unique $w$-subgroup of $F$. It is well-known that the inclusion $\langle u\rangle /\langle w\rangle \rightarrow F /\langle w\rangle\rangle$ is injective [LS01, Proposition II.5.17].

So when $\pi(w)=1$, a $w$-subgroup is unique and malnormal. In fact, malnormality holds in general. 
Lemma 6.3. If $K \leq F$ is a w-subgroup then $K$ is malnormal. In particular, if $w^{g} \in K$ then $g \in K$.

Proof. Let $g \in F$; then $K \leq\langle K, g\rangle$ and $\operatorname{rk}(\langle K, g\rangle) \leq \operatorname{rk}(K)+1$. If $k_{1}^{g}=k_{2}$ for $k_{1}, k_{2} \in K \backslash 1$ then there is a non-trivial relation between $K$ and $g$ and so, since free groups are Hopfian, $\operatorname{rk}(\langle K, g\rangle) \leq \operatorname{rk}(K)$. Therefore, by the definition of a $w$-subgroup, $\langle K, g\rangle=K$, so $g \in K$.

Uniqueness in the case $\pi(w)=1$ extends to finiteness in general, and the finite list of $w$-subgroups is computable. Here we deduce computability from Whitehead's algorithm [Whi36; an alternative algorithm is described in Pud14, Appendix A].

Lemma 6.4. There are only finitely many $w$-subgroups in a free group $F$, and there is an algorithm that lists them.

Proof. If $F$ is the fundamental group of a based finite graph $\Omega$, then any finitely generated subgroup $K$ can be realized by a based immersion of finite core graphs $\Lambda \leftrightarrow \Omega$, and if $w$ is contained in $K$ then the immersion $w: S \rightarrow \Omega$ lifts to $\Lambda$. We only need to consider subgroups $K$ for which $w$ is not contained in a proper free factor, and for such subgroups $K$, every edge of $\Lambda$ is in the image of $w$. Unless $w$ is primitive in $K$, every edge of $\Lambda$ is hit at least twice by $w$, so we only need to consider the finitely many based immersions $\Lambda \rightarrow \Omega$ with $|\Lambda| \leq|w| / 2$. For each such $\Lambda \rightarrow \Omega$, Whitehead's algorithm decides whether or not $w$ is primitive in $K$. Keep those $\Lambda$ of minimal rank, and of these the $w$-subgroups are the maximal ones with respect to inclusion: $K \leq K^{\prime}$ if and only if the based immersion $\Lambda \rightarrow \Omega$ factors through the based immersion $\Lambda^{\prime} \rightarrow \Omega$, which can be checked trivially.

If we realize $F$ as the fundamental group of a core graph $\Omega$ and $w$ by an immersion $w: S \rightarrow \Omega$ then each of the finitely many $w$-subgroups $K_{i}$ is realized by an immersion of core graphs $\Lambda_{i} \rightarrow \Omega$. We may then define complexes $Q_{i}=$ $\Lambda_{i} \cup_{w} D$ (where $w$ is the unique, by Lemma 6.3, lift of $w$ to $\Lambda_{i}$ ), which come equipped with immersions $Q_{i} \rightarrow X$. These play a key role in the classification of immersions $Y \rightarrow X$ with $\chi(Y)=2-\pi(w)$.

Definition 6.5. If $K_{i} \leq F$ is a $w$-subgroup we also call $P_{i}=K_{i} /\langle\langle w\rangle$ a $w-$ subgroup of $G=F /\langle w\rangle\rangle$.

The $w$-subgroups come equipped with homomorphisms $P_{i} \rightarrow G$ induced by the immersions $Q_{i} \rightarrow X$. The name ' $w$-subgroup' turns out to be justified, since by Theorem 6.17 these homomorphisms are injective.

Remark 6.6. Whenever a one-relator group $F /\langle w\rangle\rangle$ splits freely, the word $w$ is conjugate into a free factor of $F$ [LS01, Proposition II.5.13]. In particular, every $w$-subgroup $P_{i}=K_{i} /\langle\langle w\rangle$ is at most one-ended, since $w$ is not contained in a proper free factor of $K_{i}$. (Note that $\mathbb{Z}$ is a HNN extension of the trivial group, and in particular splits freely.) 


\subsection{Nielsen reduction}

This section introduces the strong version of homotopy equivalence that plays a role in our main results.

Definition 6.7. Let $X, X^{\prime}$ be combinatorial 2-complexes. A Nielsen equivalence between $X$ and $X^{\prime}$ is a homotopy equivalence $f: G_{X} \rightarrow G_{X^{\prime}}$ and a homeomorphism $s: S_{X} \rightarrow S_{X^{\prime}}$ such that $f \circ w_{X} \simeq w_{X^{\prime}} \circ s$. (Here, we use the notation of Definition 2.1.) In this case, we say that $X$ and $X^{\prime}$ are Nielsen equivalent.

Let $Y$ be another 2-complex. We say that $X$ Nielsen reduces to $Y$ if $X$ is Nielsen equivalent to a wedge $Y \vee \bigvee_{i} D_{i}^{2}$, where the $D_{i}^{2}$ are 2-discs with the standard cellular structure.

Complexes that Nielsen reduce to graphs can also be characterized algebraically. The following theorem is an easy consequence of the fact that any pair of bases of a free group are related by Nielsen moves [LS01, Proposition I.4.1].

Proposition 6.8. A two-complex $Y$ Nielsen reduces to a graph if and only if the conjugacy classes represented by the attaching maps for the two-cells of $Y$ have representatives which are a sub-basis of the free group $\pi_{1}\left(Y^{(1)}\right)$.

We will make use of the following technical fact about Nielsen reduction. It is an immediate consequence of Proposition 6.8, because the pullback of a sub-basis along an immersion is a sub-basis.

Lemma 6.9. Let $U, Y$ be 2-complexes. If $U$ immerses in $Y$ and $Y$ Nielsen reduces to a graph, then $U$ Nielsen reduces to a graph. In particular, if $Y$ is one-relator and the attaching map is along a primitive element, then $U$ Nielsen reduces to a graph.

\subsection{One-relator pushouts and primitivity rank}

We can now classify immersions of finite complexes $Y \rightarrow X$ when $\chi(Y)$ is sufficiently large: specifically, when $\chi(Y) \geq 2-\pi(w)$.

Lemma 6.10. Let $G=F /\langle w\rangle$ be a one-relator group as above, and $X$ a presentation complex of $G$, with $w$ represented by an immersion $w: S \rightarrow \Omega$. Let $Y \rightarrow X$ be an immersion from a compact connected one- or two-complex $Y$ to $X$. Suppose that $\chi(Y) \geq 2-\pi(w)$, that $Y$ has no free faces, and that the one-skeleton of $Y$ is a core graph.

(i) If $\chi(Y)>2-\pi(w)$ then $Y$ reduces to a graph.

(ii) If $\chi(Y)=2-\pi(w)$ then either $Y$ reduces to a graph or $Y \rightarrow X$ factors through some $Q_{i} \rightarrow X$. 
Proof. Since $Y$ has no free faces, Corollary 3.2 implies that $\chi\left(\hat{Y}^{I}\right) \geq \chi(Y)$, where $\hat{Y}^{I}$ is the immersed one-relator pushout of $Y$ (Definition 3.1.)

We first prove item (i). Suppose that $\chi(Y)>2-\pi(w)$. If $\pi_{1}\left(\Gamma_{W}^{I}\right)$ is the subgroup of $F$ corresponding to the 1-skeleton of $\hat{Y}^{I}$,

$$
\operatorname{rk}\left(\pi_{1}\left(\Gamma_{W}^{I}\right)\right)=2-\chi\left(\hat{Y}^{I}\right) \leq 2-\chi(Y)<\pi(w) .
$$

Since $\pi_{1}\left(\Gamma_{W}^{I}\right)$ is a subgroup of $F$ of rank less than $\pi(w), w$ represents a primitive element of $\pi_{1}\left(\Gamma_{W}^{I}\right)$, so $Y$ reduces to a graph, by Lemma 6.9 .

The proof of item (ii) is similar. If $Y$ is a graph there is nothing to prove. If $w$ is primitive in $\pi_{1}\left(\Gamma_{W}^{I}\right)$ then, as in the previous paragraph, $Y$ reduces to a graph. Otherwise, $\operatorname{rk}\left(\pi_{1}\left(\Gamma_{W}^{I}\right)\right)=\pi(w)$ and $w$ is not primitive in $\pi_{1}\left(\Gamma_{W}^{I}\right)$, so there is a $w$-subgroup $K_{i}$ of $F$ containing $\pi_{1}\left(\Gamma_{W}^{I}\right)$. Since $Y^{(1)}$ is a core graph, $\Gamma_{W}^{I}$ is also a core graph, and so the immersion $\Gamma_{W}^{I} \rightarrow \Omega$ factors through $Q_{i}^{(1)}=\Lambda_{i}$. Therefore $\hat{Y}^{I} \rightarrow X$ factors through $Q_{i} \rightarrow X$, and so $Y \rightarrow X$ also factors through $Q_{i}$.

\subsection{Homomorphisms from finitely generated groups}

In this section we combine the observations from the previous subsections and finally prove Theorem 1.5 The first lemma provides a tool for promoting results about immersions to results about subgroups.

Lemma 6.11. A combinatorial map of finite 2-complexes $X \rightarrow Y$ factors as

$$
X \rightarrow Z \leftrightarrow Y
$$

where $X \rightarrow Z$ is surjective and $\pi_{1}$-surjective.

Proof. This is part of [LWar, Lemma 4.1].

This has the following useful consequence.

Lemma 6.12. Let $Y$ be a finite 2-complex, and let $f: H \rightarrow \pi_{1}(Y)$ be a homomorphism from a finitely presented group. Then there is a an immersion from a finite, connected 2-complex $g: Z \rightarrow Y$ and a surjection $h: H \rightarrow \pi_{1}(Z)$ such that $f=g_{*} \circ h$.

Proof. Let $\left\langle x_{1}, \ldots, x_{m} \mid r_{1}, \ldots, r_{n}\right\rangle$ be a finite presentation for $H$. Let $R \rightarrow$ $Y$ be a combinatorial map from a rose $R$ with petals corresponding to the $x_{i}$. Each relator $r_{j}$ is the boundary of a singular disc diagram $D_{j} \rightarrow Y$. Let $X$ be constructed by gluing the $D_{j}$ to $R$ along their boundaries. There is a combinatorial map $X \rightarrow Y$ realizing the homomorphism $f$. Applying Lemma 6.11. $X \rightarrow Y$ factors through an immersion $Z \rightarrow Y$.

For homomorphisms from finitely generated groups, we obtain the following, slightly weaker, result. 
Lemma 6.13. Let $Y$ be a finite 2-complex, and let $f: H \rightarrow \pi_{1}(Y)$ be a homomorphism from an n-generator group. There is a sequence of $\pi_{1}$-surjective immersions of finite, connected 2-complexes without free faces

$$
Z_{0} \rightarrow Z_{1} \rightarrow \cdots \leftrightarrow Z_{i} \rightarrow \cdots,
$$

an immersion $g$ from the direct limit $Z=\lim _{i} Z_{i}$ into $Y$ and a $\pi_{1}$-surjection $h: H \rightarrow \pi_{1}(Z)$ such that $f=g_{*} \circ h$. Furthermore, we may take $\operatorname{rk}\left(\pi_{1}\left(Z_{0}\right)\right) \leq n$.

Proof. The existence of the sequence of immersions is [LWar, Lemma 4.4], and the final assertion about $\operatorname{rk}\left(\pi_{1}\left(Z_{0}\right)\right)$ is an immediate consequence of its proof.

In general, when one applies Lemma 6.11 there may be no relation between the Euler characteristics of the complexes $X$ and $Z$. However, we will obtain some control using a theorem of Howie. Recall that a group is locally indicable if every non-trivial finitely generated subgroup has infinite abelianization.

Theorem ([How81, Corollary 4.2]). If $X$ is a 2-complex and $Y \subseteq X$ is a connected subcomplex such that $\pi_{1}(Y)$ is locally indicable and $H_{2}(X, Y)=0$ then the map $\pi_{1}(Y) \rightarrow \pi_{1}(X)$ induced by inclusion is injective.

We use Howie's theorem to prove the following lemma, which can also be deduced from earlier results of Stallings [Sta65, p171].

Lemma 6.14. If $X$ is a connected 2-complex and $\pi_{1}(X)$ is generated by $n$ elements, then

$$
\chi(X) \geq 1-n
$$

with equality only if $\pi_{1}(X)$ is free on $n$ generators.

Proof. Let $x_{1}, \ldots, x_{n}$ be a generating set for $\pi_{1}(X)$. Since $X$ is 2-dimensional and $b_{1}(X) \leq n$ it is clear that $\chi(X) \geq 1-n$, so it suffices to show $\pi_{1}(X)$ is free on the $x_{i}$ if $\chi(X)=1-n$. We can realize the $x_{i}$ by a combinatorial $\pi_{1}$-surjection of a rose $f: R \rightarrow X$. Let $M$ be the mapping cylinder of $f$, a 2-complex homotopy-equivalent to $X$. If $\chi(M)=1-n$ then $H_{2}(M)=0$ and the natural map $H_{1}(R) \rightarrow H_{1}(M)$ is injective. Therefore, by the long exact sequence of a pair, $H_{2}(M, R)=0$ and so by Howie's theorem, $\pi_{1}(R) \rightarrow \pi_{1}(M)$ is injective, since free groups are locally indicable. Therefore, $\pi_{1}(M)=\pi_{1}(X)$ is free on the $x_{i}$.

Remark 6.15. Lemma 6.14 quickly implies a classical result of Magnus [Mag39. In the one-relator case (which Magnus attributes to Dehn), the result is as follows: if $\left.G=F_{n} /\langle w\rangle\right\rangle$, then either $G \cong F_{n-1}$ or $\operatorname{rk}(G)=n$ [LS01, Proposition II.5.11].

Lemma 6.14 also enables us to prove the group-theoretic analogue of Lemma 6.10 . from which Theorem 1.5 follows immediately.

Lemma 6.16. Let $G=F /\langle w\rangle\rangle$ be a one-relator group with $\pi(w)>1$, and let $f: H \rightarrow G$ be a homomorphism from a finitely generated group $H$. 
(i) If $\operatorname{rk}(H)<\pi(w)$ then $f$ factors through a free group.

(ii) If $\operatorname{rk}(H)=\pi(w)$ and $H$ is not free of rank $\pi(w)$ then either $f$ factors through a free group or $f(H)$ is conjugate into some $w$-subgroup $P_{k}$.

Proof. By Lemma 6.13, there is a sequence of $\pi_{1}$-surjective immersions of finite, connected 2-complexes without free faces

$$
Z_{0} \leftrightarrow Z_{1} \leftrightarrow \cdots \leftrightarrow Z_{i} \rightarrow \cdots
$$

so that $f$ factors through $\pi_{1}(Z)$, where $Z=\underline{\lim } Z_{i}$. Therefore, if $f$ does not factor through a free group, $\pi_{1}(Z)$ is not free. Since free groups are Hopfian, $\pi_{1}\left(Z_{i}\right)$ is not free for all but finitely many $i$, and so we may assume without loss of generality that $\pi_{1}\left(Z_{i}\right)$ is not free for any $i$.

If $\operatorname{rk}(H)<\pi(w)$ then, for all $i$,

$$
\chi\left(Z_{i}\right) \geq 2-\operatorname{rk}(H)>2-\pi(w)
$$

by Lemma 6.14 and so $Z_{i}$ Nielsen reduces to a graph by Lemma 6.10, which contradicts the assumption that $\pi_{1}\left(Z_{i}\right)$ is not free. This proves item (i).

If $\operatorname{rk}(H)=\pi(w)$ then, similarly, $\chi\left(Z_{i}\right) \geq 2-\pi(w)$ for all $i$, and since $\pi_{1}\left(Z_{i}\right)$ is not free, we must have $\chi\left(Z_{i}\right)=2-\pi(w)$. Therefore, by Lemma 6.10 each immersion $Z_{i} \rightarrow X$ factors through some $Q_{k(i)} \rightarrow X$. Since there are only finitely many $Q_{k}$ by Lemma 6.4, there is a $k$ such that $Z_{i} \rightarrow X$ factors through $Q_{k}$ for infinitely many $i$, whence $f$ factors through $P_{k}$. This proves item (ii).

\section{5 w-subgroups are subgroups}

At last we can prove, as claimed, that the $w$-subgroups $P_{i}$ really are subgroups of the one-relator group $G$. Recall that the maps $P_{i} \rightarrow G$ are induced by immersions of one-relator complexes $Q_{i} \rightarrow X$.

Theorem 6.17. Let $F$ be a free group with $w \in F$. The natural maps $P_{i} \rightarrow G$ are injective.

Proof. We assume that $w$ is nontrivial and that $\pi(w)>1$, since the case $\pi(w)=$ 1 is well-known, as noted in Example 6.2.

Let $\gamma: S^{1} \rightarrow Q_{i}$ be an edge loop whose image in $X$ is null-homotopic. Let $D$ be a van Kampen diagram for $\gamma$. Let $R=Q_{i} \cup_{\gamma} D$, which comes equipped with a natural map $R \rightarrow X$. By Lemma 6.11, this factors as

$$
R \rightarrow Z \rightarrow X
$$

with $R \rightarrow Z$ a $\pi_{1}$-surjection; in particular, we obtain a $\pi_{1}$-surjection $Q_{i} \rightarrow Z$. The complex $Z$ retracts to a subcomplex $Y \subseteq Z$ without free faces, and since $Q_{i}$ has no free faces the immersion $Q_{i} \rightarrow X$ factors through the retraction to $Y$. Now, $H=\pi_{1}(Y)$ is generated by $\pi(w)$ elements and is not free of rank $\pi(w)$ since it is a quotient of $P_{i}$, so by Lemma 6.14 $\chi(Y) \geq 2-\pi(w)$. Therefore, by Lemma 6.10, either $Y$ reduces to a graph or it factors through some immersion 
$Q_{j} \rightarrow X$. But the immersion $Q_{i} \rightarrow X$ factors through the immersion $Q_{i} \rightarrow Y$, so by Lemma 6.9 if $Y$ reduces to a graph then $Q_{i}$ does too, contradicting the definition of a $w$-subgroup. Therefore $Y \rightarrow X$ factors through some $Q_{j}$. It follows that $K_{i} \leq K_{j}$ (where these are the $w$-subgroups of $F$ corresponding to $Q_{i}$ and $Q_{j}$ respectively) so, by the definition of a $w$-subgroup, $i=j$ and $Q_{i} \rightarrow Q_{j}$ is an isomorphism. Therefore, $R$ retracts to $Q_{i}$, so $\gamma$ was already null-homotopic in $Q_{i}$. This proves the theorem.

Using Remark 6.6, we see that $\pi(w)$ is an invariant of the isomorphism type of the one-relator group $G$.

Corollary 6.18. If $w \in F$ is a word in a free group then $\pi(w)$ is the minimal rank of a non-free subgroup of the one-relator group $G=F /\langle\langle w\rangle$.

\subsection{The case $\pi(w)=2$}

As explained in the introduction, the results of the previous section show that, when $\pi(w)>2$, the subgroup structure of $G=F /\langle\langle w\rangle$ is like the subgroup structure of a hyperbolic group. In this section, we examine the case $\pi(w)=2$, and notice that the non-negatively curved behaviour of $G$ is concentrated in a particular subgroup. This follows from the next result, which shows that in this case there is a unique $w$-subgroup of $F$.

Proposition 6.19. Let $F$ be a free group and $w \in F \backslash 1$ an imprimitive element that is not a proper power. If $H_{1}$ and $H_{2}$ are rank-two subgroups of $F$ with $w$ contained in, but not primitive in, both $H_{1}$ and $H_{2}$, then $\left\langle H_{1}, H_{2}\right\rangle$ also has rank two.

$$
\text { If } \pi(w)=2 \text { then there is a unique } w \text {-subgroup of } F \text {. }
$$

Proof. Since $w$ is imprimitive and not a proper power, Theorem 1.16 applies to give

$$
1 \leq\left(\operatorname{rk}\left(H_{1}\right)-1\right)+\left(\operatorname{rk}\left(H_{2}\right)-1\right)-\left(\operatorname{rk}\left(\left\langle H_{1}, H_{2}\right\rangle\right)-1\right),
$$

and since $\operatorname{rk}\left(H_{1}\right)=\operatorname{rk}\left(H_{2}\right)=2$, it follows that $\operatorname{rk}\left(\left\langle H_{1}, H_{2}\right\rangle\right)=2$ as required.

Suppose that $\pi(w)=2$. Let $\mathcal{H}=\left\{H_{i}\right\}$ be the set of rank-two subgroups of $F$ so that $w \in H_{i}$ and $w$ is not primitive in $H_{i} ; \mathcal{H}$ is finite by Lemma 6.4 and since $\pi(w)=2, \mathcal{H}$ is non-empty. Considering the partial order on $\mathcal{H}$ given by inclusion, the previous paragraph now implies that each pair has an upper bound, and it follows that $\mathcal{H}$ has a unique maximal element $K$, which is necessarily the unique $w$-subgroup.

Therefore, in this case, we drop the unnecessary subscript $i$ and write $P$ for the $w$-subgroup of $G$. In light of Conjecture 1.12 we make the following definition.

Definition 6.20. If $\pi(w)=2$ then $P$ is the peripheral subgroup of $G$. 
We do not currently know how to prove that $P$ is uniquely defined in $G$ up to isomorphism. However, Lemma 6.16 shows that if $G \cong F /\langle w\rangle \cong F^{\prime} /\left\langle w^{\prime}\right\rangle$ are isomorphic then the corresponding peripheral subgroups $P$ and $P^{\prime}$ are conjugate into each other, which somewhat justifies the term 'peripheral'. If Conjecture 1.12 held then $P$ would be malnormal in $G$, and therefore would be a well-defined isomorphism invariant.

\section{References}

[AO96] G. N. Arzhantseva and A. Yu. Ol'shanskiu, Generality of the class of groups in which subgroups with a lesser number of generators are free, Mat. Zametki 59 (1996), no. 4, 489-496, 638. MR 1445193

[Bau60] Gilbert Baumslag, Some aspects of groups with unique roots, Acta Math. 104 (1960), 217-303.

[Bau65] _ Residual nilpotence and relations in free groups, J. Algebra 2 (1965), 271-282.

[Bau69] _ A non-cyclic one-relator group all of whose finite quotients are cyclic, J. Austral. Math. Soc. 10 (1969), 497-498.

[Bes] Mladen Bestvina, Questions in geometric group theory, http://www. math.utah.edu/ bestvina/eprints/questions-updated.pdf.

[BF05] M. Bestvina and M. Feighn, Counting maps from a surface to a graph, Geom. Funct. Anal. 15 (2005), no. 5, 939-961.

[BW99] Martin R. Bridson and Daniel T. Wise, $\mathscr{V} \mathscr{H}$ complexes, towers and subgroups of $F \times F$, Mathematical Proceedings of the Cambridge Philosophical Society 126 (1999), no. 3, 481-497.

[CH20] Christopher H. Cashen and Charlotte Hoffmann, Short, highly imprimitive words yield hyperbolic one-relator groups, arXiv:2006.15923v1 [math.GR], 2020.

[Coc54] W. H. Cockcroft, On two-dimensional aspherical complexes, Proc. London Math. Soc. (3) 4 (1954), 375-384. MR 0063042

[Cul81] Marc Culler, Using surfaces to solve equations in free groups, Topology 20 (1981), no. 2, 133-145.

[Deh11] M. Dehn, Über unendliche diskontinuierliche Gruppen, Math. Ann. 71 (1911), no. 1, 116-144.

[DH91] Andrew J. Duncan and James Howie, The genus problem for onerelator products of locally indicable groups, Math. Z. 208 (1991), no. 2, 225-237. 
[Dic94] Warren Dicks, Equivalence of the strengthened Hanna Neumann conjecture and the amalgamated graph conjecture, Invent. Math. 117 (1994), no. 3, 373-389.

[GW19] Giles Gardam and Daniel J. Woodhouse, The geometry of one-relator groups satisfying a polynomial isoperimetric inequality, Proc. Amer. Math. Soc. 147 (2019), no. 1, 125-129. MR 3876736

[How81] James Howie, On pairs of 2-complexes and systems of equations over groups, J. Reine Angew. Math. 324 (1981), 165-174.

[HW01] G. Christopher Hruska and Daniel T. Wise, Towers, ladders and the B. B. Newman spelling theorem, J. Aust. Math. Soc. 71 (2001), no. 1, $53-69$.

[HW16] Joseph Helfer and Daniel T. Wise, Counting cycles in labeled graphs: the nonpositive immersion property for one-relator groups, Int. Math. Res. Not. IMRN (2016), no. 9, 2813-2827.

[LM09] Larsen Louder and D. B. McReynolds, Graphs of subgroups of free groups, Algebr. Geom. Topol. 9 (2009), no. 1, 327-335.

[Lou13] Larsen Louder, Scott complexity and adjoining roots to finitely generated groups, Groups Geom. Dyn. 7 (2013), no. 2, 451-474.

[LS01] R. C. Lyndon and Paul E. Schupp, Combinatorial group theory, Classics in Mathematics, Springer-Verlag, Berlin, 2001, Reprint of the 1977 edition.

[LW17] Larsen Louder and Henry Wilton, Stackings and the $W$-cycles Conjecture, Canad. Math. Bull. 60 (2017), no. 3, 604-612.

[LWar] Larsen Louder and Henry Wilton, One-relator groups with torsion are coherent, Math. Res. Lett. (to appear).

[Lyn50] R. C. Lyndon, Cohomology theory of groups with a single defining relation, Ann. of Math. (2) 52 (1950), 650-665.

[Lyn59] _ The equation $a^{2} b^{2}=c^{2}$ in free groups, Michigan Math. J 6 (1959), 89-95.

[Mag30] Wilhelm Magnus, Über diskontinuierliche Gruppen mit einer definierenden Relation. (Der Freiheitssatz), J. Reine Angew. Math. 163 (1930), 141-165.

[Mag32] W. Magnus, Das Identitätsproblem für Gruppen mit einer definierenden Relation, Math. Ann. 106 (1932), no. 1, 295-307.

[Mag39] Wilhelm Magnus, Über freie Faktorgruppen und freie Untergruppen gegebener Gruppen, Monatsh. Math. Phys. 47 (1939), no. 1, 307-313. 
[New68] B. B. Newman, Some results on one-relator groups, Bull. Amer. Math. Soc. 74 (1968), 568-571.

[OS04] A. Yu. Olshanskii and M. V. Sapir, The conjugacy problem and Higman embeddings, Mem. Amer. Math. Soc. 170 (2004), no. 804, viii+133.

[PP15] Doron Puder and Ori Parzanchevski, Measure preserving words are primitive, J. Amer. Math. Soc. 28 (2015), no. 1, 63-97.

[Pud14] Doron Puder, Primitive words, free factors and measure preservation, Israel J. Math. 201 (2014), no. 1, 25-73.

[Pud15] _ Expansion of random graphs: new proofs, new results, Invent. Math. 201 (2015), no. 3, 845-908. MR 3385636

[Sta65] John R. Stallings, Homology and central series of groups, J. Algebra 2 (1965), 170-181.

[Sta83a] _ Surfaces in three-manifolds and nonsingular equations in groups, Math. Z. 184 (1983), no. 1, 1-17.

[Sta83b] — Topology of finite graphs, Invent. Math. 71 (1983), no. 3, 551565.

[Whi36] J. H. C. Whitehead, On equivalent sets of elements in a free group, Ann. of Math. (2) 37 (1936), no. 4, 782-800. MR 1503309

[Wis18] Daniel T. Wise, Coherence, local-indicability and non-positive immersions, Personal communication, 2018.

L. Louder, Department of Mathematics, University College London, Gower Street, LONDON WC1E 6BT, UK

E-mail address: 1.louder@ucl.ac.uk

H. Wilton, DPMms, Centre for Mathematical Sciences, Wilberforce Road, CamBRIDGE CB3 0WB, UK

E-mail address: h.wilton@maths.cam.ac.uk 